\title{
Optical solutions to the Kundu-Mukherjee-Naskar equation: mathematical and graphical analysis with oblique wave propagation
}

\author{
Dipankar Kumar ${ }^{1}$, Gour Paul ${ }^{2}$, Tapsh Biswas ${ }^{1}$, Aly Seadawy ${ }^{3}$, Rakib Baowali ${ }^{1}$, Mostafa \\ $\mathrm{Kamal}^{1}$, and Hadi Rezazadeh ${ }^{4}$ \\ ${ }^{1}$ Bangabandhu Sheikh Mujibur Rahman Science and Technology University \\ ${ }^{2}$ University of Rajshahi \\ ${ }^{3}$ Taibah University \\ ${ }^{4}$ Amol University of Special Modern Technologies
}

August 12, 2020

\begin{abstract}
This paper retrieves some new optical solutions to the Kundu-Mukherjee-Naskar (KMN) equation in the context of nonlinear optical fiber communication systems. In this regard, the generalized Kudryashov and new auxiliary equation methods are applied to the KMN equation and consequently, dark, bright, periodic U-shaped and singular soliton solutions are explored. The discrepancies between the present obtained solutions and the previously obtained solutions by using different methods are discussed. The time fractional derivative and an oblique wave transformation in coordination with the methods of interest are considered for acquiring new optical wave solutions of the KMN equation in the sense of conformable derivative and wave obliqueness, respectively. The effects of obliqueness and fractionality on the attained solutions are demonstrated graphically along with its physical descriptions. It is found that the optical wave phenomena are changed with the increase of obliqueness as well as fractionality. All the obtained optical solutions are found to be new in the sense of conformable derivative, wave obliqueness, and the applied methods. Finally, it is found that the utilized methods and the relevant transformation are powerful over the other methods and it can be applicable for further studies to explain the pragmatic phenomena in optical fiber communication systems.
\end{abstract}

\section{Introduction}

1.1 Background and literature review

To study of optical soliton is a dynamic research area in the fields of mathematical physics [1] and fiber optic communication systems [2]. It can play a vital role in the telecommunication industry to explain the soliton propagation effect in optical fibers and their impact on optical fiber communication systems. In the context of fiber optics, the relevant models can explain the propagation of soliton pulses through intercontinental distances. The dynamics of soliton propagation through nonlinear optics, optical fibers, metamaterials, and crystals are described by several model equations, such as the nonlinear unstable Schrödinger's equation [3], Sasa-Satsuma equation [4], complex Ginzburg-Landau equation [5], perturbed Gerdjikov-Ivanov equation [6], Lakshmanan-Posezian-Daniel equation [7, 8], Chen-Lee-Liu equation [9-11], Liquid crystals equation [12] and several others. It is worth mentioning that some researchers have studied a number of various known models and investigated their corresponding soliton dynamics via diverse analytical methods, viz. the Kudryashov method [13-15], the generalized Kudryashov method [16], the extended Kudryashov method [17], the trial solution method [18], the extended trial equation method [19], the modified simple equation method 
[20], the sine-Gordon expansion equation method [21, 22], the extended sinh-Gordon equation expansion method [23-25], simplest equation method [26], the extended simplest equation method [27], new extended direct algebraic method [28], new auxiliary equation expansion method [29] and so on. This paper deals with one of such models viz. the Kundu-Mukherjee-Naskar (KMN) equation, which can be applied to address optical wave propagation through coherently excited resonant waveguides in particular in the phenomena of bending of light beams [19]. It is also used to address the problems of hole waves and oceanic rogue waves [30]. The model can further find to be applicable to the study of soliton pulses occurring in $(2+1)$-dimensional equations [31]. The most important feature of this model is that it has been given as a new extension of nonlinear Schrödinger (NLS) equation with the inclusion of different forms of nonlinearity with regard to Kerr and non-Kerr law nonlinearities to study soliton pulses in $(2+1)$-dimensions [31, 32]. Recently, solitons in KMN equation have been addressed by several researchers to recover some optical solitons using trial equation technique [33], extended trial function method [19], ansatz approach and sine Gordon expansion method [34], F-expansion and functional variable principle [35], new extended algebraic method [36], the method of undetermined coefficients and Lie symmetry [37], modified simple equation approach [20,38] and first integral method [39]. As a result, investigators have reported some new optical solutions such as dark, bright, singular type soliton solutions. However, no studies have been found to investigate the optical solutions to the KMN equation by using the generalized Kudryashov method (gKM) and new auxiliary equation method (NAEM).

\subsection{Objective of the study}

The aim of this study is to adopt the gKM and newly developed NAEM to secure some new optical solutions, namely dark, bright, periodic U-shaped and singular soliton solutions to the KMN equation that can be of great importance in the field of fiber optics and optical communications. Furthermore, our intension is to implement the conformable derivative and wave oblique complex transform in coordination with the mentioned methods of interest to the KMN equation for obtaining new optical solutions in the sense of fractional derivative and wave obliqueness.

\subsection{Governing equation}

The dimensionless form of $(2+1)$-dimensional KMN equation is [33-38]

$i Q_{t}+p Q_{\mathrm{xy}}+i q Q\left(Q Q_{x}^{*}-Q^{*} Q_{x}\right)=0 .(1.1)$

The KMN equation specified by (1.1) was introduced by Kundu et al. [30], which is a new extension of the well-known NLS equation. In Eq. (1.1), $x$ and $y$ stand for the spatial variables while $t$ designates the temporal variable. The dependent variable $Q(x, y, t)$ represents nonlinear wave envelope, where the asterisk denotes the complex conjugate of $Q$. The first term in Eq. (1.1) stands for denoting the temporal evolution of the wave followed by the dispersion term that is given by the coefficient of $p$. The constant $q$ ensures the existence of the different case of nonlinearity media which does not fall into the conventional Kerr law nonlinearity or any known non-Kerr law media [31]. The nonlinear term in this equation accounts for "current-like" nonlinearity that stems from chirality [33].

\subsection{Application of complex transformation to the KMN equation}

In order to get optical solutions of Eq. (1.1), the following transformation is selected [33-38]

$Q(x, y, t)=U(\xi) e^{i \eta(x, y, t)}$

where $U(\xi)$ represents the amplitude component with $\xi=l_{1} x+l_{2} y$ vt, the phase component $\eta(x, y, t)$ of the soliton is defined $\operatorname{as} \eta(x, y, t)=-h_{1} x-h_{2} y+\omega t+\theta_{0}$ with $i=\sqrt{-1}$. Here, $h_{1}$ and $h_{2}$ refer to the frequencies of the soliton in the $x$ - and $y$-directions, respectively, while $\omega$ and $\theta_{0}$, respectively, correspond to the wave number and phase of the soliton. Also, the parameters $l_{1}$ and $l_{2}$ in Eq. (1.2) represent the inverse width of the soliton along the $x$ and y directions, respectively, while $\mathrm{v}$ represents the velocity of the soliton. Plugging the above transformation into KMN equation specified by Eq. (1.1) and decomposing into real and imaginary parts, the following pair of equations are attained: 
$\alpha l_{1} l_{2} U^{\prime \prime}-\left(\omega+\alpha h_{1} h_{2}\right) U 2 \beta h_{1} U^{3}=0(1.3)$

and $v=-p\left(l_{1} h_{2}+l_{2} h_{1}\right),(1.4)$

where a prime denotes the derivative with respect to $\xi$.

\subsection{Arrangement of the study}

The rest part of the paper is organized as follows. Section 2 deals with the overview of the gKM and NAUM. Applications of these methods and two important remarks are presented in Section 3. The graphical analysis of the obtained solutions is elaborated in Section 4. Finally, a general discussion and conclusion are placed in Section 5.

\section{Overview of the methods}

In this section, we will recount the workflow of the gKM and NAEM to investigate some new optical solutions to a nonlinear evolution equation (NLEE).

Assume a general (2+1)-dimensional NLEE in the following form:

$P\left(Q, Q_{x}, Q_{y}, Q_{t}, Q_{\mathrm{xx}}, Q_{\mathrm{xy}}, Q_{\mathrm{xt}}, Q_{\mathrm{yt}}, Q_{\mathrm{tt}}, Q_{\mathrm{xtt}}, \ldots \ldots\right)=0,(2.1)$

where $Q=Q(x, y, t)$ is an unknown function of complex-valued, $P$ is a polynomial of $Q(x, y, t)$, and its various partial derivatives, in which the linear and nonlinear partial derivatives are involved.

With the introduction of the transformation $Q(x, y, t)=U(\xi) e^{i \eta(x, y, t)}$, where $\xi=l_{1} x+l_{2} y v t$, and $\eta(x, y, t)=-h_{1} x-h_{2} y+\omega t+\theta_{0}$, Eq. (2.1) is converted to the following nonlinear ordinary differential equation (ODE):

$O\left(U, U^{\prime}, U^{\prime \prime} \ldots \ldots \ldots\right)=0,(2.2)$

where $O$ is a polynomial of $U$ and its derivatives, and the superscripts indicate the total derivatives with respect to $\xi$.

2.1. Overview of the generalized Kudryashov method (gKM)

The main steps of the gKM are as follows [16]:

Let us assume that the solution $U(\xi)$ of the nonlinear Eq. (2.2) can be presented as

$U(\xi)=\frac{\sum_{i=0}^{N} a_{i} R^{i}(\xi)}{\sum_{j=0}^{M} b_{j} R^{j}(\xi)}$.

In Eq. (2.3), the constants $a_{i}(i=0,1,2 \ldots, N) \operatorname{and} b_{j}(j=0,1,2 \ldots, M)$ are to be determined later. $M$ and $N$ are positive integers which can be computed by means of homogenous balance principle, and $R(\xi)$ satisfies the following ODE:

$R^{\prime}(\xi)=\left(R^{2}(\xi)-R(\xi)\right) \ln (a) \cdot(2.4)$

Equation (2.4) yields the exact solution $R(\xi)=\frac{1}{\left(1+d a^{\xi}\right)}$, where $a \neq 0,1$ and $d \neq 0$.

Substituting Eq. (2.3) along with Eq. (2.4) into Eq. (2.2) and using some mathematical operations, we get a system of algebraic equations. Solving the attained system and setting the obtained values in Eq. (2.3), one can produce exact solutions of Eq. (2.1).

2.2. Overview of the new auxiliary equation method (NAEM)

The main outlines of the NAEM are as follows [29]:

Let us assume that the solution $U(\xi)$ of the nonlinear Eq. (2.2) can be presented as

$U(\xi)=\sum_{i=0}^{N} a_{i}\left(a^{f(\xi)}\right)^{i},(2.5)$ 
where the constant coefficients $a_{i}(i=0,1,2 \ldots, N)$ are to be determined and $N$ is a positive integer which can be computed by means of homogenous balance principle. For Eq. $(2.5), f(\xi)$ satisfies the following ODE: $f^{\prime}(\xi)=\frac{1}{\ln (a)}\left(\alpha a^{-f(\xi)}+\beta+\sigma a^{f(\xi)}\right)$.

Equation (2.6) yields the following family of solutions:

Family-I: When $\beta^{2}-4 \alpha \sigma<0$ and $\sigma \neq 0$,

$a^{f(\xi)}=-\frac{\beta}{2 \sigma}+\frac{\sqrt{4 \alpha \sigma-\beta^{2}}}{2 \sigma} \tan \left(\frac{\sqrt{4 \alpha \sigma-\beta^{2}}}{2} \xi\right)$,
$a^{f(\xi)}=-\frac{\beta}{2 \sigma}-\frac{\sqrt{4 \alpha \sigma-\beta^{2}}}{2 \sigma} \cot \left(\frac{\sqrt{4 \alpha \sigma-\beta^{2}}}{2} \xi\right)$.

Family-II: When $\beta^{2}-4 \alpha \sigma>0$ and $\sigma \neq 0$,

$a^{f(\xi)}=-\frac{\beta}{2 \sigma}-\frac{\sqrt{\beta^{2}-4 \alpha \sigma}}{2 \sigma} \tanh \left(\frac{\sqrt{\beta^{2}-4 \alpha \sigma}}{2} \xi\right)$,

$a^{f(\xi)}=-\frac{\beta}{2 \sigma}-\frac{\sqrt{\beta^{2}-4 \alpha \sigma}}{2 \sigma} \operatorname{coth}\left(\frac{\sqrt{\beta^{2}-4 \alpha \sigma}}{2} \xi\right)$.

Family-III: When $\beta^{2}+4 \alpha^{2}<0, \sigma=-\alpha$ and $\sigma \neq 0$,

$a^{f(\xi)}=\frac{\beta}{2 \sigma}-\frac{\sqrt{-\left(\beta^{2}+4 \alpha^{2}\right)}}{2 \sigma} \tan \left(\frac{\sqrt{-\left(\beta^{2}+4 \alpha^{2}\right)}}{2} \xi\right)$,
$a^{f(\xi)}=\frac{\beta}{2 \sigma}+\frac{\sqrt{-\left(\beta^{2}+4 \alpha^{2}\right)}}{2 \sigma} \cot \left(\frac{\sqrt{-\left(\beta^{2}+4 \alpha^{2}\right)}}{2} \xi\right)$.

Family-IV: When $\beta^{2}+4 \alpha^{2}>0, \sigma=-\alpha$ and $\sigma \neq 0$,

$a^{f(\xi)}=\frac{\beta}{2 \sigma}-\frac{\sqrt{\left(\beta^{2}+4 \alpha^{2}\right)}}{2 \sigma} \tanh \left(\frac{\sqrt{\left(\beta^{2}+4 \alpha^{2}\right)}}{2} \xi\right)$,

$a^{f(\xi)}=\frac{\beta}{2 \sigma}+\frac{\sqrt{\left(\beta^{2}+4 \alpha^{2}\right)}}{2 \sigma} \cot \left(\frac{\sqrt{\left(\beta^{2}+4 \alpha^{2}\right)}}{2} \xi\right)$.

Family-V: When $\beta^{2}-4 \alpha^{2}<0$ and $\sigma=\alpha$,

$a^{f(\xi)}=-\frac{\beta}{2 \sigma}+\frac{\sqrt{-\left(\beta^{2}-4 \alpha^{2}\right)}}{2 \sigma} \tan \left(\frac{\sqrt{-\left(\beta^{2}-4 \alpha^{2}\right)}}{2} \xi\right)$,

$a^{f(\xi)}=-\frac{\beta}{2 \sigma}+\frac{\sqrt{-\left(\beta^{2}-4 \alpha^{2}\right)}}{2 \sigma} \cot \left(\frac{\sqrt{-\left(\beta^{2}-4 \alpha^{2}\right)}}{2} \xi\right)$.

Family-VI: When $\beta^{2}-4 \alpha^{2}>0$ and $\sigma=\alpha$,

$a^{f(\xi)}=-\frac{\beta}{2 \sigma}-\frac{\sqrt{\left(\beta^{2}-4 \alpha^{2}\right)}}{2 \sigma} \tanh \left(\frac{\sqrt{\left(\beta^{2}-4 \alpha^{2}\right)}}{2} \xi\right)$,

$a^{f(\xi)}=-\frac{\beta}{2 \sigma}-\frac{\sqrt{\left(\beta^{2}-4 \alpha^{2}\right)}}{2 \sigma} \operatorname{coth}\left(\frac{\sqrt{\left(\beta^{2}-4 \alpha^{2}\right)}}{2} \xi\right)$.

Family-VII: When $\alpha \sigma>0, \beta=0$ and $\sigma \neq 0$,

$a^{f(\xi)}=\sqrt{\frac{\alpha}{\sigma}} \tan (\sqrt{\alpha \sigma} \xi)$,

$a^{f(\xi)}=\sqrt{\frac{\alpha}{\sigma}} \cot (\sqrt{\alpha \sigma} \xi)$.

Family-VIII: When $\alpha \sigma<0, \beta=0$ and $\sigma \neq 0$, 
$a^{f(\xi)}=\sqrt{-\frac{\alpha}{\sigma}} \tanh (\sqrt{-\alpha \sigma} \xi)$,

$a^{f(\xi)}=\sqrt{-\frac{\alpha}{\sigma}} \operatorname{coth}(\sqrt{-\alpha \sigma} \xi)$.

Family-IX: When $\beta^{2}-4 \alpha \sigma=0$,

$a^{f(\xi)}=\frac{-2 \alpha(\beta \xi+2)}{\beta^{2} \xi}$.

Family-X: When $\beta=0$ and $\alpha=-\sigma$,

$a^{f(\xi)}=\frac{e^{2 \alpha \xi}+1}{e^{2 \alpha \xi}-1}$.

Family-XI: When $\alpha=\sigma=0$,

$a^{f(\xi)}=-\frac{e^{2 \beta \xi}+1}{2 e^{\beta \xi}}$.

Family-XII: When $\alpha=2 K, \beta=K$ and $\sigma=0$,

$a^{f(\xi)}=e^{\mathrm{K} \xi}-2$.

$\boldsymbol{\Phi} \alpha \mu \iota \lambda \psi$ - $\boldsymbol{\Xi I I I}: \Omega \eta \varepsilon \nu \sigma=K, \beta=K \alpha \nu \delta \alpha=0$,

$a^{f(\xi)}=\frac{e^{\mathrm{K}}}{1-e^{\mathrm{K}}}$.

$\Phi \alpha \mu \iota \lambda \psi$ - $\Xi I I I: ~ \Omega \eta \varepsilon \nu \alpha=0$,

$a^{f(\xi)}=\frac{\beta e}{2-\sigma e}$.

$\boldsymbol{\Phi} \alpha \mu \iota \lambda \psi-\boldsymbol{\Xi} \mathbf{I}^{*}: \Omega \eta \varepsilon \nu \beta=\sigma=0$,

$a^{f(\xi)}=\alpha \xi$.

$\Phi \alpha \mu \iota \lambda \psi-\Xi^{\prime \prime}: \Omega \eta \varepsilon \nu \beta=\alpha=0$,

$a^{f(\xi)}=-\frac{1}{\sigma \xi}$.

$\Phi \alpha \mu \iota \lambda \psi-\Xi^{*} \mathbf{I}: \Omega \eta \varepsilon \nu \beta=0 \alpha \nu \delta \alpha=\sigma$,

$a^{f(\xi)}=\tan (\alpha \xi+E)$.

$\Phi \alpha \mu \iota \lambda \psi-\Xi^{*} \mathbf{I I}: \Omega \eta \varepsilon \nu \sigma=0$,

$a^{f(\xi)}=e^{\beta \xi}-\frac{\alpha}{\beta}$.

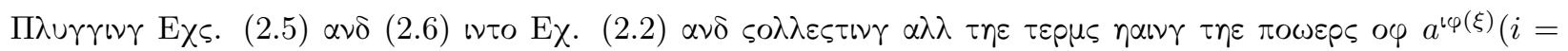

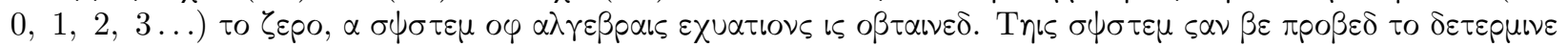

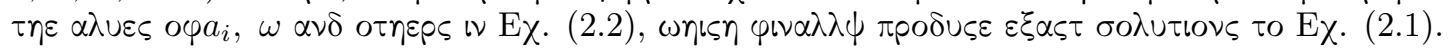

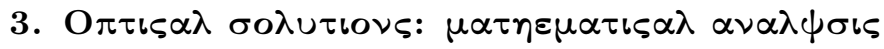

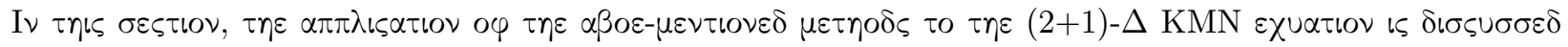

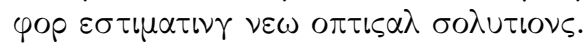

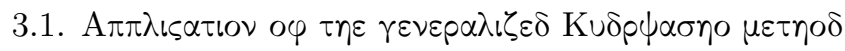

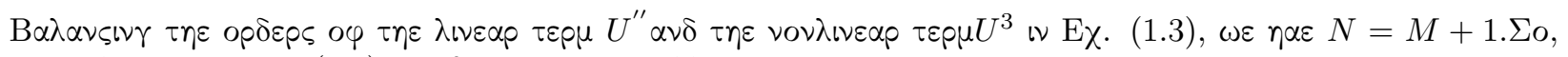

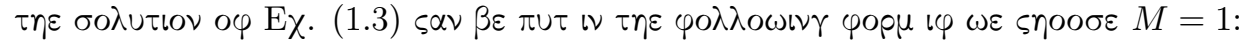

$U(\xi)=\frac{a_{0}+a_{1} R(\xi)+a_{2} R^{2}(\xi)}{b_{0}+b_{1} R(\xi)}$.

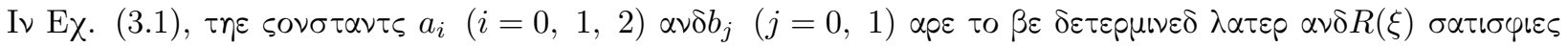
$\tau \eta \varepsilon \mathrm{O} \Delta \mathrm{E} \sigma \pi \varepsilon \varsigma \iota \varphi \iota \varepsilon \delta \beta \psi \mathrm{E} \chi$. . (2.2). 


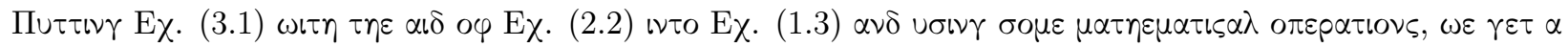

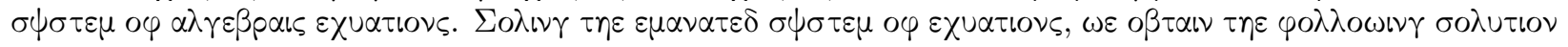

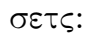

$\Sigma \varepsilon \tau-\mathrm{I}: a_{0}=0, a_{1}=\mp \frac{1}{2} \frac{p b_{1} l_{1} l_{2} \lambda \nu(a)}{\sqrt{\pi \chi h_{1} l_{1} l_{2}}}, a_{2}= \pm b_{1} \ln (a) \sqrt{\frac{p l_{1} l_{2}}{q h_{1}}}, b_{0}=0$,

$\omega=-\frac{1}{2} p\left(l_{1} l_{2}(\ln (a))^{2}+2 h_{1} h_{2}\right), \alpha \nu \delta b_{1}$ i $\varphi p \varepsilon \varepsilon$.

$\Sigma \varepsilon \tau$-II: $a_{0}=0, a_{1}= \pm 2 b_{0} \ln (a) \sqrt{\frac{p l_{1} l_{2}}{q h_{1}}}, a_{2}=\mp 2 b_{0} \ln (a) \sqrt{\frac{p l_{1} l_{2}}{q h_{1}}}, b_{1}=-2 b_{0}$,

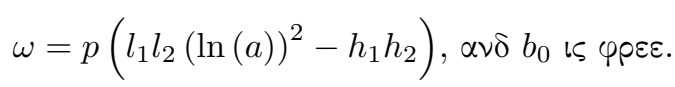

$\Sigma \varepsilon \tau$-III: $a_{0}=0, a_{1}= \pm \frac{b_{1} \ln (a)}{2} \frac{\sqrt{\pi \times h_{1} l_{1} l_{2}}}{q h_{1}}, a_{2}=\mp \frac{p l_{1} l_{2} \ln (a) b_{1}}{\sqrt{\pi \times h_{1} l_{1} l_{2}}}, b_{0}=0$,

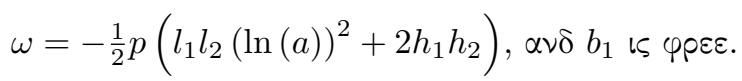

$\Sigma \varepsilon \tau-I^{\prime \prime}: a_{0}= \pm \frac{b_{0} \ln (a) \sqrt{\pi \times h_{1} l_{1} l_{2}}}{q h_{1}}, a_{1}=\mp \frac{2 b_{0} \ln (a) \sqrt{\pi \times h_{1} l_{1} l_{2}}}{q h_{1}}, a_{2}= \pm \frac{2 p l_{1} l_{2} b_{0} \ln (a)}{\sqrt{\pi \times h_{1} l_{1} l_{2}}}, b_{1}=-2 b_{0}, \omega=$

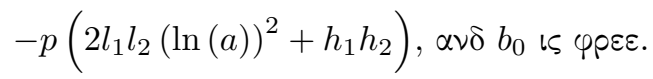

$\Sigma \varepsilon \tau-": a_{0}= \pm \frac{1}{2} \frac{b_{0} \ln (a) \sqrt{\pi \times h_{1} l_{1} l_{2}}}{q h_{1}}, a_{1}=0, a_{2}=\mp \frac{2 p l_{1} l_{2} b_{0} \ln (a)}{\sqrt{\pi \times h_{1} l_{1} l_{2}}}, b_{1}=2 b_{0}$,

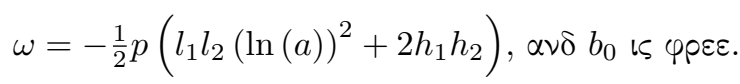

$\Sigma \varepsilon \tau-{ }^{\circ} \mathrm{I}: a_{2}=-\left(4 a_{0}+2 a_{1}\right), b_{0}= \pm \frac{2 q h_{1} a_{0}}{\ln (a) \sqrt{\pi \times h_{1} l_{1} l_{2}}}, b_{1}= \pm \frac{2 \sqrt{\pi \chi h_{1} l_{1} l_{2}}\left(2 a_{0}+a_{1}\right)}{p l_{1} l_{2} \ln (a)}$,

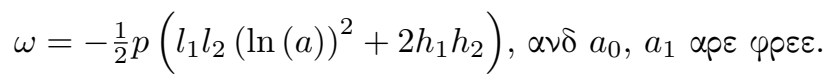

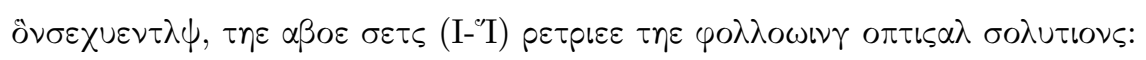

$$
\begin{aligned}
& Q_{1,2}(x, y, t)=\frac{\mp\left(\frac{1}{2} \frac{p b_{1} l_{1} l_{2} \lambda \nu(a)}{\sqrt{\pi \times h_{1} l_{1} l_{2}}} \frac{1}{(1+d a \xi)}-b_{1} \ln (a) \sqrt{\frac{p l_{1} l_{2}}{h_{1}}} \frac{1}{(1+d a \xi)^{2}}\right)}{\frac{b_{1}}{(1+d a \xi)}} \times e^{i\left(-h_{1} x-h_{2} \psi-\frac{1}{2} p\left(l_{1} l_{2}(\ln (a))^{2}+2 h_{1} h_{2}\right) t+\theta_{0}\right)}, \\
& Q_{3,4}(x, y, t)=\frac{ \pm 2 b_{0} \ln (a) \sqrt{\frac{p l_{1} l_{2}}{q h_{1}}}\left(\frac{1}{(1+d a \xi)}-\frac{1}{(1+d a \xi)^{2}}\right)}{b_{0}\left(1-\frac{2}{(1+d a \xi)}\right)} \times e^{i\left(-h_{1} x-h_{2} y+p\left(l_{1} l_{2}(\ln (a))^{2}-h_{1} h_{2}\right) t+\theta_{0}\right)}, \\
& Q_{5,6}(x, y, t)=\frac{ \pm\left(\frac{b_{1} \ln (a)}{2} \frac{\sqrt{\pi x h_{1} l_{1} l_{2}}}{h_{1} h_{1}} \frac{1}{(1+d a \xi)}-\frac{p l_{1} l_{2} \ln (a) b_{1}}{\sqrt{\pi \times h_{1} l_{1} l_{2}}} \frac{1}{(1+d a \xi)^{2}}\right)}{\left(\frac{b_{1}}{(1+d a \xi)}\right)} \times e^{i\left(-h_{1} x-h_{2} y-\frac{p}{2}\left(l_{1} l_{2}(\ln (a))^{2}+2 h_{1} h_{2}\right) t+\theta_{0}\right)}, \\
& Q_{7,8}(x, y, t) \quad=\quad \frac{ \pm\left(\frac{b_{0} \ln (a) \sqrt{\pi \times h_{1} l_{1} l_{2}}}{q h_{1}}-\frac{2 b_{0} \ln (a) \sqrt{\pi \times h_{1} l_{1} l_{2}}}{q h_{1}} \frac{1}{(1+d a \xi)}+\frac{2 p l_{1} l_{2} b_{0} \ln (a)}{\sqrt{\pi \times h_{1} l_{1} l_{2}}} \frac{1}{(1+d a \xi)^{2}}\right)}{b_{0}\left(1-\frac{2}{(1+d a \xi}\right)} \times \\
& e^{i\left(-h_{1} x-h_{2} \psi-p\left(2 l_{1} l_{2}(\ln (a))^{2}+h_{1} h_{2}\right) t+\theta_{0}\right)}, \\
& Q_{9,10}(x, y, t)=\frac{ \pm\left(\frac{1}{2} \frac{b_{0} \ln (a) \sqrt{\pi \times h_{1} l_{1} l_{2}}}{q h_{1}}-\frac{2 p l_{1} l_{2} b_{0} \ln (a)}{\sqrt{\pi \times h_{1} l_{1} l_{2}}} \frac{1}{(1+d a \xi}\right)}{b_{0}\left(1+\frac{2}{(1+d a \xi)}\right)} \times e^{i\left(-h_{1} x-h_{2} \psi-\frac{1}{2} p\left(l_{1} l_{2}(\ln (a))^{2}+2 h_{1} h_{2}\right) t+\theta_{0}\right)}
\end{aligned}
$$


$Q_{11,12}(x, y, t)=\frac{a_{0}+\frac{a_{1}}{(1+d a \xi)}-\frac{\left(4 a_{0}+2 a_{1}\right)}{(1+d a \xi)^{2}}}{ \pm\left(\frac{2 q h_{1} a_{0}}{\ln (a) \sqrt{\pi \times h_{1} l_{1} l_{2}}}+\frac{2 \sqrt{\pi \times h_{1} l_{1} l_{2}\left(2 a_{0}+a_{1}\right)}}{p l_{1} l_{2} \ln (a)} \frac{1}{(1+d a \xi)}\right)} \times e^{i\left(-h_{1} x-h_{2} \psi-\frac{h_{1}}{b_{1}^{2}}\left(p b_{1}^{2} h_{2}+2 q a_{1}^{2}\right) t+\theta_{0}\right)}$,

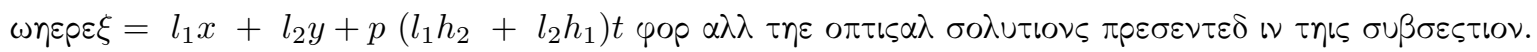

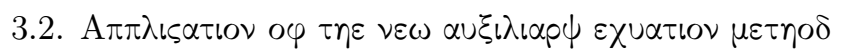

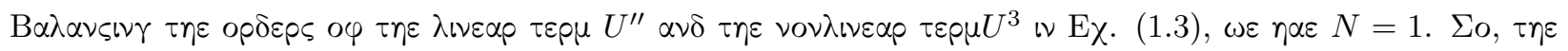

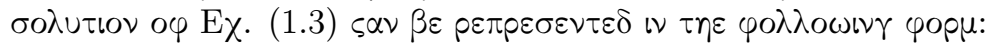

$U(\xi)=a_{0}+a_{1} a^{f(\xi)},(3.2)$

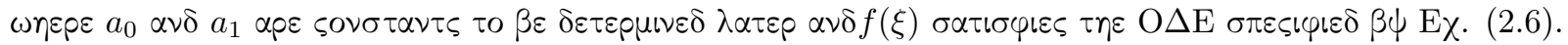

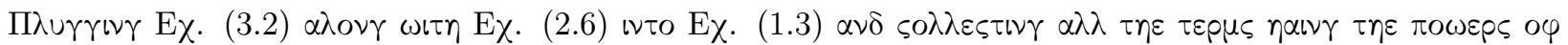

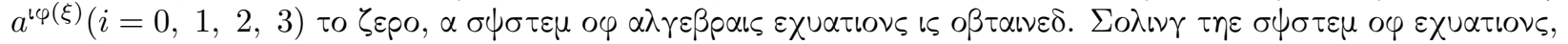

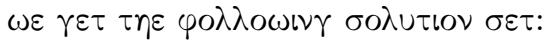

$a_{0}= \pm \frac{\beta}{2 q h_{1}} \sqrt{\pi \chi h_{1} l_{1} l_{2}}, a_{1}= \pm \frac{\sigma}{q h_{1}} \sqrt{\pi \chi h_{1} l_{1} l_{2}} \alpha \nu \delta \omega=\frac{1}{2} p\left(4 \alpha \sigma l_{1} l_{2}-\beta^{2} l_{1} l_{2}-2 h_{1} h_{2}\right)$.

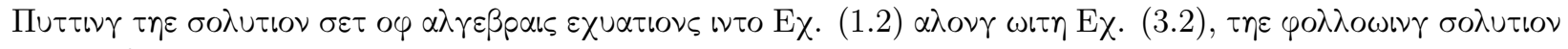

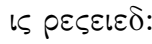

$Q(x, y, t)= \pm \frac{1}{2 q h_{1}} \sqrt{\pi \chi h_{1} l_{1} l_{2}}\left(\beta+2 \sigma a^{f(\xi)}\right) \times e^{i\left(-h_{1} x-h_{2} y+\left(\frac{p}{2}\left(4 \alpha \sigma l_{1} l_{2}-\beta^{2} l_{1} l_{2}-2 h_{1} h_{2}\right)\right) t+\theta_{0}\right)}$,

$\omega \eta \varepsilon p \varepsilon \xi=l_{1} x+l_{2} y+p\left(l_{1} h_{2}+l_{2} h_{1}\right) t$.

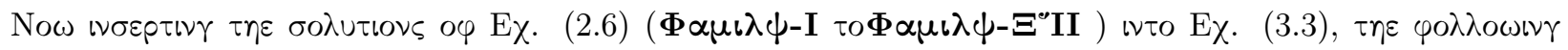

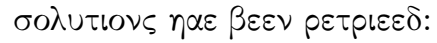

Фор $\boldsymbol{\Phi} \alpha \mu \iota \lambda \psi$-I: $\Omega \eta \varepsilon \nu \beta^{2}-4 \alpha \sigma<0 \alpha \nu \delta \sigma \neq 0$,

$Q_{1,2}(x, y, t) \quad=\quad \pm \frac{1}{2 q h_{1}} \sqrt{\pi \chi h_{1} l_{1} l_{2}}\left(\beta+2 \sigma\left(-\frac{\beta}{2 \sigma}+\frac{\sqrt{4 \alpha \sigma-\beta^{2}}}{2 \sigma} \tan \left(\frac{\sqrt{4 \alpha \sigma-\beta^{2}}}{2} \xi\right)\right)\right) \times$ $e^{i\left(-h_{1} x-h_{2} y+\left(\frac{p}{2}\left(4 \alpha \sigma l_{1} l_{2}-\beta^{2} l_{1} l_{2}-2 h_{1} h_{2}\right)\right) t+\theta_{0}\right)}$,

$Q_{3,4}(x, y, t) \quad=\quad \pm \frac{1}{2 q h_{1}} \sqrt{\pi \chi h_{1} l_{1} l_{2}}\left(\beta+2 \sigma\left(-\frac{\beta}{2 \sigma}-\frac{\sqrt{4 \alpha \sigma-\beta^{2}}}{2 \sigma} \cot \left(\frac{\sqrt{4 \alpha \sigma-\beta^{2}}}{2} \xi\right)\right)\right) \times$ $e^{i\left(-h_{1} x-h_{2} y+\left(\frac{p}{2}\left(4 \alpha \sigma l_{1} l_{2}-\beta^{2} l_{1} l_{2}-2 h_{1} h_{2}\right)\right) t+\theta_{0}\right)}$.

Фop $\Phi \alpha \mu \iota \lambda \psi$-II: $\Omega \eta \varepsilon \nu \beta^{2}-4 \alpha \sigma>0 \alpha \nu \delta \sigma \neq 0$,

$Q_{5,6}(x, y, t) \quad=\quad \pm \frac{1}{2 q h_{1}} \sqrt{\pi \chi h_{1} l_{1} l_{2}}\left(\beta+2 \sigma\left(-\frac{\beta}{2 \sigma}-\frac{\sqrt{\beta^{2}-4 \alpha \sigma}}{2 \sigma} \tanh \left(\frac{\sqrt{\beta^{2}-4 \alpha \sigma}}{2} \xi\right)\right)\right) \times$ $e^{i\left(-h_{1} x-h_{2} y+\left(\frac{p}{2}\left(4 \alpha \sigma l_{1} l_{2}-\beta^{2} l_{1} l_{2}-2 h_{1} h_{2}\right)\right) t+\theta_{0}\right)}$,

$Q_{7,8}(x, y, t) \quad=\quad \pm \frac{1}{2 q h_{1}} \sqrt{\pi \chi h_{1} l_{1} l_{2}}\left(\beta+2 \sigma\left(-\frac{\beta}{2 \sigma}-\frac{\sqrt{\beta^{2}-4 \alpha \sigma}}{2 \sigma} \operatorname{coth}\left(\frac{\sqrt{\beta^{2}-4 \alpha \sigma}}{2} \xi\right)\right)\right) \times$ $e^{i\left(-h_{1} x-h_{2} y+\left(\frac{p}{2}\left(4 \alpha \sigma l_{1} l_{2}-\beta^{2} l_{1} l_{2}-2 h_{1} h_{2}\right)\right) t+\theta_{0}\right)}$.

Фop $\mathbf{\Phi} \alpha \mu \iota \lambda \psi$-III: $\Omega \eta \varepsilon \nu \beta^{2}+4 \alpha^{2}<0, \sigma=-\alpha \alpha \nu \delta \sigma \neq 0$,

$Q_{9,10}(x, y, t) \quad=\quad \pm \frac{1}{2 q h_{1}} \sqrt{\pi \chi h_{1} l_{1} l_{2}}\left(\beta+2 \sigma\left(\frac{\beta}{2 \sigma}-\frac{\sqrt{-\left(\beta^{2}+4 \alpha^{2}\right)}}{2 \sigma} \tan \left(\frac{\sqrt{-\left(\beta^{2}+4 \alpha^{2}\right)}}{2} \xi\right)\right)\right) \times$ $e^{i\left(-h_{1} x-h_{2} y+\left(\frac{p}{2}\left(4 \alpha \sigma l_{1} l_{2}-\beta^{2} l_{1} l_{2}-2 h_{1} h_{2}\right)\right) t+\theta_{0}\right)}$,

$Q_{11,12}(x, y, t) \quad=\quad \pm \quad \frac{1}{2 q h_{1}} \sqrt{\pi \chi h_{1} l_{1} l_{2}}\left(\beta+2 \sigma\left(\frac{\beta}{2 \sigma}+\frac{\sqrt{-\left(\beta^{2}+4 \alpha^{2}\right)}}{2 \sigma} \cot \left(\frac{\sqrt{-\left(\beta^{2}+4 \alpha^{2}\right)}}{2} \xi\right)\right)\right) \times$ $e^{i\left(-h_{1} x-h_{2} y+\left(\frac{p}{2}\left(4 \alpha \sigma l_{1} l_{2}-\beta^{2} l_{1} l_{2}-2 h_{1} h_{2}\right)\right) t+\theta_{0}\right)}$. 


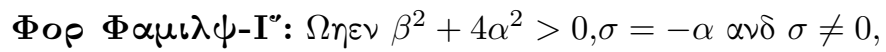

$Q_{13,14}(x, y, t) \quad=\quad \pm \frac{1}{2 q h_{1}} \sqrt{\pi \chi h_{1} l_{1} l_{2}}\left(\beta+2 \sigma\left(\frac{\beta}{2 \sigma}-\frac{\sqrt{\left(\beta^{2}+4 \alpha^{2}\right)}}{2 \sigma} \tanh \left(\frac{\sqrt{\left(\beta^{2}+4 \alpha^{2}\right)}}{2} \xi\right)\right)\right) \times$ $e^{i\left(-h_{1} x-h_{2} y+\left(\frac{p}{2}\left(4 \alpha \sigma l_{1} l_{2}-\beta^{2} l_{1} l_{2}-2 h_{1} h_{2}\right)\right) t+\theta_{0}\right)}$,

$Q_{15,16}(x, y, t) \quad=\quad \pm \frac{1}{2 q h_{1}} \sqrt{\pi \chi h_{1} l_{1} l_{2}}\left(\beta+2 \sigma\left(\frac{\beta}{2 \sigma}+\frac{\sqrt{\left(\beta^{2}+4 \alpha^{2}\right)}}{2 \sigma} \cot \left(\frac{\sqrt{\left(\beta^{2}+4 \alpha^{2}\right)}}{2} \xi\right)\right)\right) \times$ $e^{i\left(-h_{1} x-h_{2} y+\left(\frac{p}{2}\left(4 \alpha \sigma l_{1} l_{2}-\beta^{2} l_{1} l_{2}-2 h_{1} h_{2}\right)\right) t+\theta_{0}\right)}$.

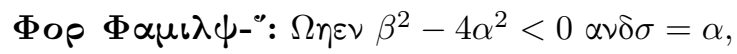

$Q_{17,18}(x, y, t) \quad=\quad \pm \frac{1}{2 q h_{1}} \sqrt{\pi \chi h_{1} l_{1} l_{2}}\left(\beta+2 \sigma\left(-\frac{\beta}{2 \sigma}+\frac{\sqrt{-\left(\beta^{2}-4 \alpha^{2}\right)}}{2 \sigma} \tan \left(\frac{\sqrt{-\left(\beta^{2}-4 \alpha^{2}\right)}}{2} \xi\right)\right)\right) \times$ $e^{i\left(-h_{1} x-h_{2} y+\left(\frac{p}{2}\left(4 \alpha \sigma l_{1} l_{2}-\beta^{2} l_{1} l_{2}-2 h_{1} h_{2}\right)\right) t+\theta_{0}\right)}$,

$Q_{19,20}(x, y, t) \quad=\quad \pm \quad \frac{1}{2 q h_{1}} \sqrt{\pi \chi h_{1} l_{1} l_{2}}\left(\beta+2 \sigma\left(-\frac{\beta}{2 \sigma}+\frac{\sqrt{-\left(\beta^{2}-4 \alpha^{2}\right)}}{2 \sigma} \cot \left(\frac{\sqrt{-\left(\beta^{2}-4 \alpha^{2}\right)}}{2} \xi\right)\right)\right) \times$ $e^{i\left(-h_{1} x-h_{2} y+\left(\frac{p}{2}\left(4 \alpha \sigma l_{1} l_{2}-\beta^{2} l_{1} l_{2}-2 h_{1} h_{2}\right)\right) t+\theta_{0}\right)}$.

Фор $\boldsymbol{\Phi} \alpha \mu \iota \lambda \psi$ - ${ }^{\circ} \mathbf{I}: \Omega \eta \varepsilon \nu \beta^{2}-4 \alpha^{2}>0 \alpha \nu \delta \sigma=\alpha$,

$Q_{21,22}(x, y, t) \quad=\quad \pm \frac{1}{2 q h_{1}} \sqrt{\pi \chi h_{1} l_{1} l_{2}}\left(\beta+2 \sigma\left(-\frac{\beta}{2 \sigma}-\frac{\sqrt{\left(\beta^{2}-4 \alpha^{2}\right)}}{2 \sigma} \tanh \left(\frac{\sqrt{\left(\beta^{2}-4 \alpha^{2}\right)}}{2} \xi\right)\right)\right) \times$ $e^{i\left(-h_{1} x-h_{2} y+\left(\frac{p}{2}\left(4 \alpha \sigma l_{1} l_{2}-\beta^{2} l_{1} l_{2}-2 h_{1} h_{2}\right)\right) t+\theta_{0}\right)}$,

$Q_{23,24}(x, y, t) \quad=\quad \pm \frac{1}{2 q h_{1}} \sqrt{\pi \chi h_{1} l_{1} l_{2}}\left(\beta+2 \sigma\left(-\frac{\beta}{2 \sigma}-\frac{\sqrt{\left(\beta^{2}-4 \alpha^{2}\right)}}{2 \sigma} \operatorname{coth}\left(\frac{\sqrt{\left(\beta^{2}-4 \alpha^{2}\right)}}{2} \xi\right)\right)\right) \times$ $e^{i\left(-h_{1} x-h_{2} y+\left(\frac{p}{2}\left(4 \alpha \sigma l_{1} l_{2}-\beta^{2} l_{1} l_{2}-2 h_{1} h_{2}\right)\right) t+\theta_{0}\right)}$.

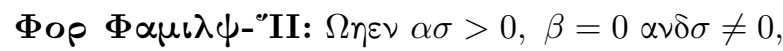

$Q_{25,26}(x, y, t)= \pm \frac{1}{2 q h_{1}} \sqrt{\pi \chi h_{1} l_{1} l_{2}}\left(\beta+2 \sigma\left(\sqrt{\frac{\alpha}{\sigma}} \tan (\sqrt{\alpha \sigma} \xi)\right)\right) \times e^{i\left(-h_{1} x-h_{2} y+\left(\frac{p}{2}\left(4 \alpha \sigma l_{1} l_{2}-\beta^{2} l_{1} l_{2}-2 h_{1} h_{2}\right)\right) t+\theta_{0}\right)}$, $Q_{27,28}(x, y, t)= \pm \frac{1}{2 q h_{1}} \sqrt{\pi \chi h_{1} l_{1} l_{2}}\left(\beta+2 \sigma\left(\sqrt{\frac{\alpha}{\sigma}} \cot (\sqrt{\alpha \sigma} \xi)\right)\right) \times e^{i\left(-h_{1} x-h_{2} y+\left(\frac{p}{2}\left(4 \alpha \sigma l_{1} l_{2}-\beta^{2} l_{1} l_{2}-2 h_{1} h_{2}\right)\right) t+\theta_{0}\right)}$.

Фop $\mathbf{\Phi} \alpha \mu \iota \lambda \psi-{ }^{\circ}$ III: $\Omega \eta \varepsilon \nu \alpha \sigma<0, \beta=0 \alpha \nu \delta \sigma \neq 0$,

$Q_{29,30}(x, y, t) \quad=\quad \pm \quad \frac{1}{2 q h_{1}} \sqrt{\pi \chi h_{1} l_{1} l_{2}}\left(\beta+2 \sigma\left(\sqrt{-\frac{\alpha}{\sigma}} \tanh (\sqrt{-\alpha \sigma} \xi)\right)\right) \quad \times$

$e^{i\left(-h_{1} x-h_{2} y+\left(\frac{p}{2}\left(4 \alpha \sigma l_{1} l_{2}-\beta^{2} l_{1} l_{2}-2 h_{1} h_{2}\right)\right) t+\theta_{0}\right)}$,

$Q_{31,32}(x, y, t) \quad=\quad \pm \quad \frac{1}{2 q h_{1}} \sqrt{\pi \chi h_{1} l_{1} l_{2}}\left(\beta+2 \sigma\left(\sqrt{-\frac{\alpha}{\sigma}} \operatorname{coth}(\sqrt{-\alpha \sigma} \xi)\right)\right) \quad \times$ $e^{i\left(-h_{1} x-h_{2} y+\left(\frac{p}{2}\left(4 \alpha \sigma l_{1} l_{2}-\beta^{2} l_{1} l_{2}-2 h_{1} h_{2}\right)\right) t+\theta_{0}\right)}$.

$\mathbf{\Phi o p} \boldsymbol{\Phi} \alpha \mu \iota \lambda \psi$-I $\Xi: \Omega \eta \varepsilon \nu \beta^{2}-4 \alpha \sigma=0$,

$Q_{33,34}(x, y, t)= \pm \frac{1}{2 q h_{1}} \sqrt{\pi \chi h_{1} l_{1} l_{2}}\left(\beta+2 \sigma\left(\frac{-2 \alpha(\beta \xi+2)}{\beta^{2} \xi}\right)\right) \times e^{i\left(-h_{1} x-h_{2} y+\left(\frac{p}{2}\left(4 \alpha \sigma l_{1} l_{2}-\beta^{2} l_{1} l_{2}-2 h_{1} h_{2}\right)\right) t+\theta_{0}\right)}$.

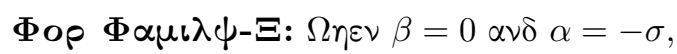

$Q_{35,36}(x, y, t)= \pm \frac{1}{2 q h_{1}} \sqrt{\pi \chi h_{1} l_{1} l_{2}}\left(\beta+2 \sigma\left(\frac{e^{2 \alpha \xi}+1}{e^{2 \alpha \xi}-1}\right)\right) \times e^{i\left(-h_{1} x-h_{2} y+\left(\frac{p}{2}\left(4 \alpha \sigma l_{1} l_{2}-\beta^{2} l_{1} l_{2}-2 h_{1} h_{2}\right)\right) t+\theta_{0}\right)}$.

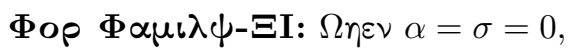

$Q_{37,38}(x, y, t)= \pm \frac{1}{2 q h_{1}} \sqrt{\pi \chi h_{1} l_{1} l_{2}}\left(\beta-2 \sigma\left(\frac{e^{2 \beta \xi}+1}{2 e^{\beta \xi}}\right)\right) \times e^{i\left(-h_{1} x-h_{2} y+\left(\frac{p}{2}\left(4 \alpha \sigma l_{1} l_{2}-\beta^{2} l_{1} l_{2}-2 h_{1} h_{2}\right)\right) t+\theta_{0}\right)}$.

Фор $\boldsymbol{\Phi} \alpha \mu \iota \lambda \psi$ - $\boldsymbol{\Xi I I}: \Omega \eta \varepsilon \nu \alpha=2 K, \beta=K \alpha \nu \delta \sigma=0$, 
$Q_{39,40}(x, y, t)= \pm \frac{1}{2 q h_{1}} \sqrt{\pi \chi h_{1} l_{1} l_{2}}\left(\beta+2 \sigma\left(e^{\mathrm{K} \xi}-2\right)\right) \times e^{i\left(-h_{1} x-h_{2} y+\left(\frac{p}{2}\left(4 \alpha \sigma l_{1} l_{2}-\beta^{2} l_{1} l_{2}-2 h_{1} h_{2}\right)\right) t+\theta_{0}\right)}$.

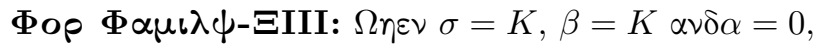

$Q_{41,42}(x, y, t)= \pm \frac{1}{2 q h_{1}} \sqrt{\pi \chi h_{1} l_{1} l_{2}}\left(\beta+2 \sigma\left(\frac{e^{\mathrm{K}}}{1-e^{\mathrm{K}}}\right)\right) \times e^{i\left(-h_{1} x-h_{2} y+\left(\frac{p}{2}\left(4 \alpha \sigma l_{1} l_{2}-\beta^{2} l_{1} l_{2}-2 h_{1} h_{2}\right)\right) t+\theta_{0}\right)}$.

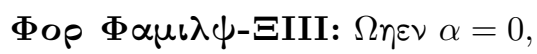

$Q_{43,44}(x, y, t)= \pm \frac{1}{2 q h_{1}} \sqrt{\pi \chi h_{1} l_{1} l_{2}}\left(\beta+2 \sigma\left(\frac{\beta e}{2-\sigma e}\right)\right) \times e^{i\left(-h_{1} x-h_{2} y+\left(\frac{p}{2}\left(4 \alpha \sigma l_{1} l_{2}-\beta^{2} l_{1} l_{2}-2 h_{1} h_{2}\right)\right) t+\theta_{0}\right)}$.

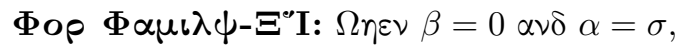

$Q_{45,46}(x, y, t)= \pm \frac{1}{2 q h_{1}} \sqrt{\pi \chi h_{1} l_{1} l_{2}}(\beta+2 \sigma(\tan (\alpha \xi+E))) \times e^{i\left(-h_{1} x-h_{2} y+\left(\frac{p}{2}\left(4 \alpha \sigma l_{1} l_{2}-\beta^{2} l_{1} l_{2}-2 h_{1} h_{2}\right)\right) t+\theta_{0}\right)}$.

Фор $\Phi \alpha \mu \iota \lambda \psi$ - $\Xi^{\circ} I I: \Omega \eta \varepsilon \nu \sigma=0$,

$Q_{47,48}(x, y, t)= \pm \frac{1}{2 q h_{1}} \sqrt{\pi \chi h_{1} l_{1} l_{2}}\left(\beta+2 \sigma\left(e^{\beta \xi}-\frac{\alpha}{\beta}\right)\right) \times e^{i\left(-h_{1} x-h_{2} y+\left(\frac{p}{2}\left(4 \alpha \sigma l_{1} l_{2}-\beta^{2} l_{1} l_{2}-2 h_{1} h_{2}\right)\right) t+\theta_{0}\right)}$.

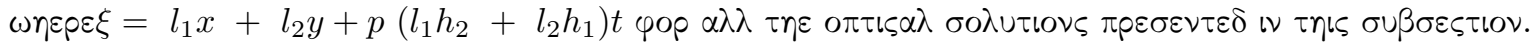

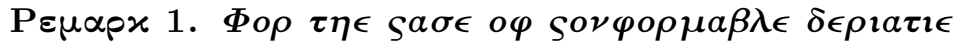

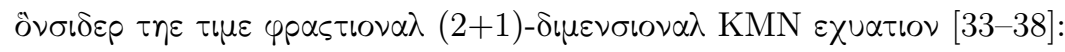

$i D_{t}^{\tau} Q+p Q_{\xi \psi}+\left\llcorner\chi \mathrm{X}\left(Q Q_{x}^{*}-Q^{*} Q_{x}\right)=0, i=\sqrt{-1},(3.4)\right.$

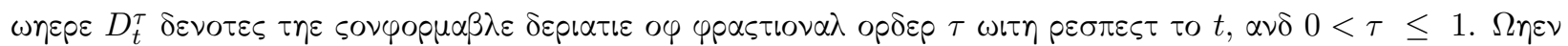

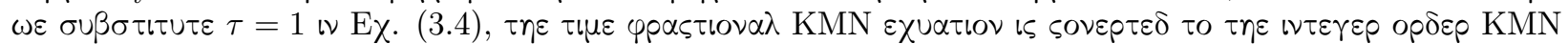

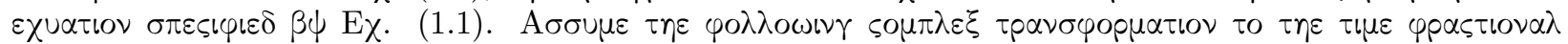

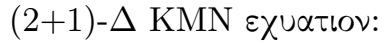

$Q(x, y, t ; \tau)=U(\xi) e^{\ln (x, y, t)}, \xi=l_{1} x+l_{2} y \quad v \frac{t^{\tau}}{\tau} \alpha \nu \delta \eta=-h_{1} x-h_{2} y+\omega \frac{t^{\tau}}{\tau}+\theta_{0}$.

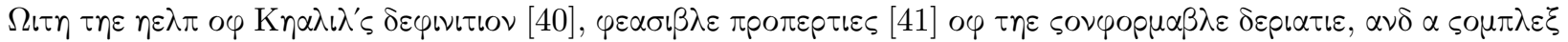

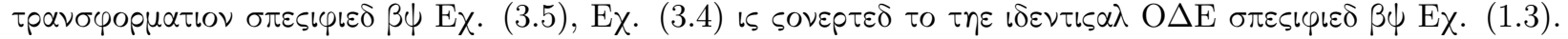

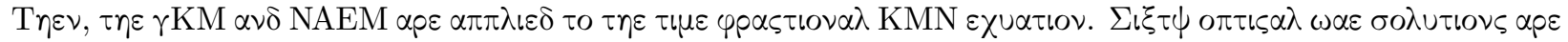

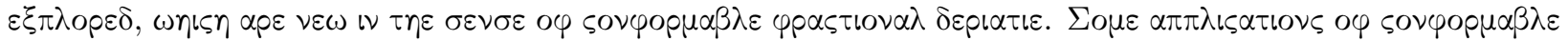

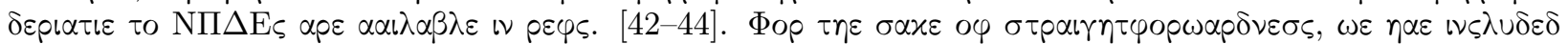

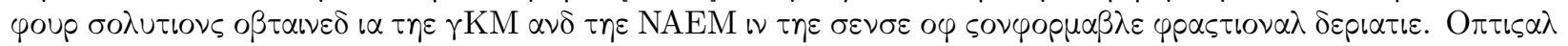

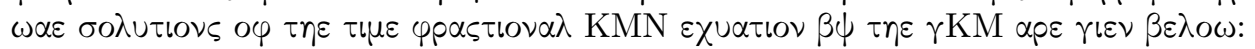

$Q_{1,2}(x, y, t ; \tau)=\frac{\mp\left(\frac{1}{2} \frac{p b_{1} l_{1} l_{2} \ln (a)}{\sqrt{\pi \times h_{1} l_{1} l_{2}}} \frac{1}{(1+d a \xi)}-b_{1} \ln (a) \sqrt{\frac{p l_{1} l_{2}}{q h_{1}}} \frac{1}{(1+d a \xi)^{2}}\right)}{\frac{b_{1}}{(1+d a \xi)}} \times e^{i\left(-h_{1} x-h_{2} y-\frac{1}{2} p\left(l_{1} l_{2}(\ln (a))^{2}+2 h_{1} h_{2}\right) \frac{t^{\tau}}{\tau}+\theta_{0}\right)}$,

$\omega \eta \varepsilon p \varepsilon \xi=l_{1} x+l_{2} y+p\left(l_{1} h_{2}+l_{2} h_{1}\right) \frac{t^{\tau}}{\tau}$.

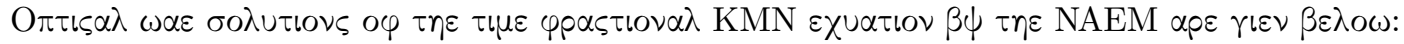

Фop $\Phi \alpha \mu \iota \lambda \psi-\mathbf{I}: \Omega \eta \varepsilon \nu \beta^{2}-4 \alpha \sigma<0 \alpha \nu \delta \sigma \neq 0$,

$Q_{1,2}(x, y, t ; \tau)= \pm \frac{1}{2 q h_{1}} \sqrt{\pi \chi h_{1} l_{1} l_{2}}\left(\beta+2 \sigma\left(-\frac{\beta}{2 \sigma}+\underline{\sqrt{4 \alpha \sigma-\beta^{2}}}\right.\right.$

$\varepsilon \vee \gamma \lambda เ \sigma \eta 2 \sigma \tan \left(\frac{\sqrt{4 \alpha \sigma-\beta^{2}}}{}\right.$

$\varepsilon \nu \gamma \lambda\lrcorner \sigma \eta 2 \xi \times e^{i\left(-h_{1} x-h_{2} y+\left(\frac{p}{2}\left(4 \alpha \sigma l_{1} l_{2}-\beta^{2} l_{1} l_{2}-2 h_{1} h_{2}\right)\right) \frac{t^{\tau}}{\tau}+\theta_{0}\right)}$,

$\omega \eta \varepsilon p \varepsilon \xi=l_{1} x+l_{2} y+p\left(l_{1} h_{2}+l_{2} h_{1}\right) \frac{t^{\tau}}{\tau}$. 


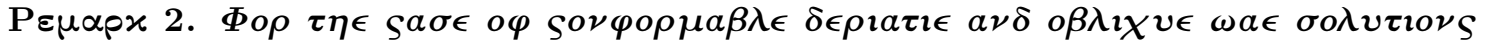

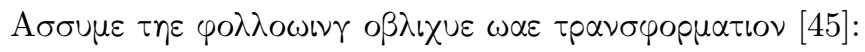

$Q(x, y, t ; \tau)=U(\xi) e^{\ln (x, y, t)},(3.8)$

$\omega \eta \varepsilon \rho \varepsilon \xi=\cos (\theta) x+\sin (\theta) y \quad v \frac{t^{\tau}}{\tau}, \alpha \nu \delta \eta=-k \cos (\theta) x-\mathrm{k} \sin (\theta) y+\omega \frac{t^{\tau}}{\tau}+\theta_{0} \omega \iota \tau \cos ^{2}(\theta)+\sin ^{2}(\theta)=1$.

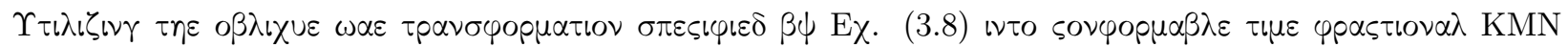

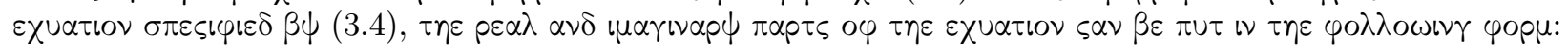

$\alpha \sin (\theta) U^{\prime \prime}-\left(\omega+\alpha k^{2} \sin (\theta)\right) U 2 \beta \chi \Upsilon^{3}=0(3.9)$

$\alpha \nu \delta=-p k \sin (2 \theta),(3.10)$

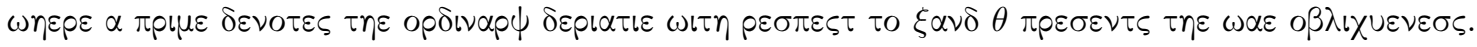

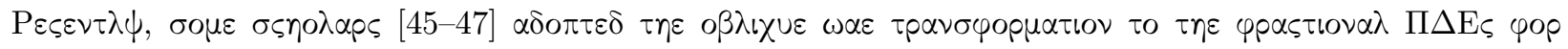

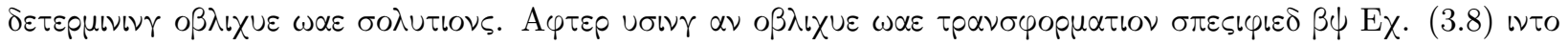

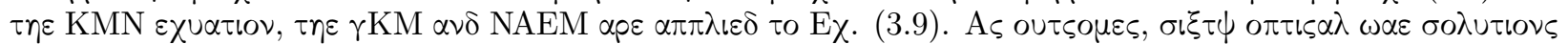

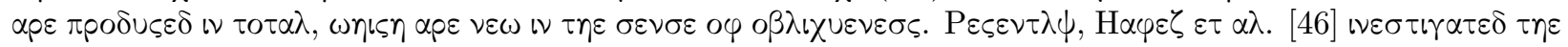

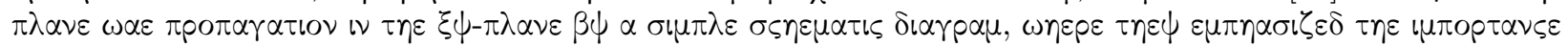
о

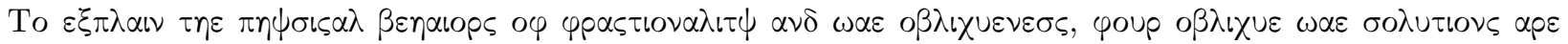

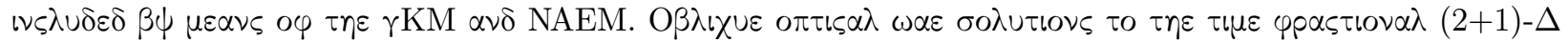

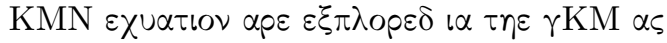

$$
\begin{aligned}
& Q_{1,2}(x, y, t ; \tau, \theta) \quad=\quad \frac{\mp\left(\frac{1}{2} \frac{p b_{1} \cos (\theta) \sin (\theta) \ln (a)}{\left.\sqrt{\pi \times \cos (\theta) \cos (\theta) \sin (\theta)} \frac{1}{(1+d a \xi)}-b_{1} \ln (a) \sqrt{\frac{p \sin (\theta)}{\chi \varkappa}} \frac{1}{\left(1+d a^{2}\right)^{2}}\right)}\right.}{\frac{b_{1}}{(1+d a \xi)}} \times \\
& e^{i\left(-\operatorname{kcos}(\theta) x-k \sin (\theta) y-\frac{1}{2} p \cos (\theta) \sin (\theta)\left((\ln (a))^{2}+2 k^{2}\right) \frac{t^{\tau}}{\tau}+\theta_{0}\right)} .(3.11)
\end{aligned}
$$

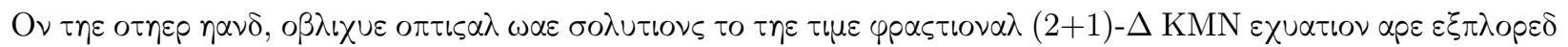

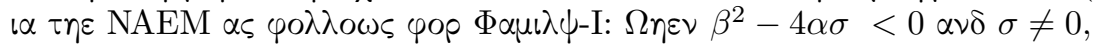

$Q_{1,2}(x, y, t ; \tau, \theta)= \pm \frac{1}{2 q \operatorname{kcos}(\theta)} \sqrt{\pi \chi \chi \cos ^{2}(\theta) \sin (\theta)}\left(\beta+2 \sigma\left(-\frac{\beta}{2 \sigma}+\frac{\sqrt{4 \alpha \sigma-\beta^{2}}}{}\right.\right.$

$\varepsilon \nu \gamma \lambda เ \sigma \eta 2 \sigma \tan \left(\frac{\sqrt{4 \alpha \sigma-\beta^{2}}}{}\right.$

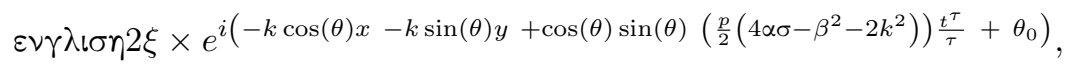

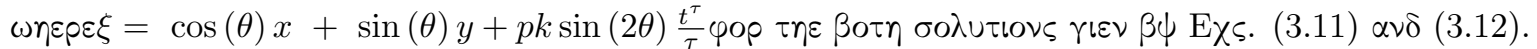

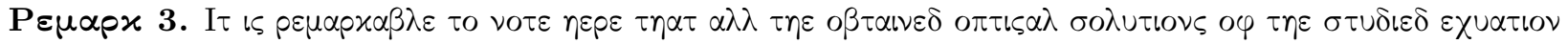

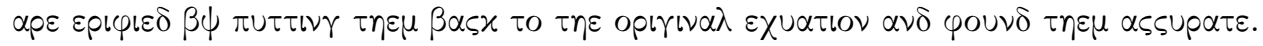

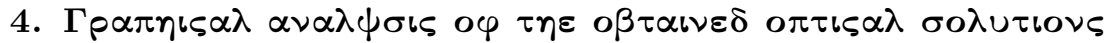

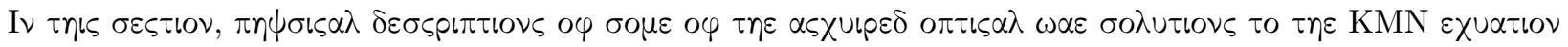

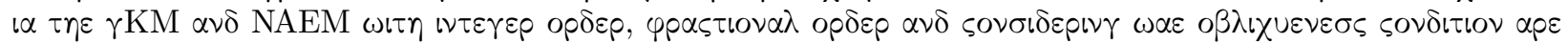

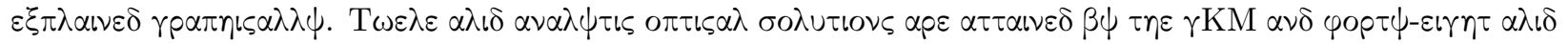

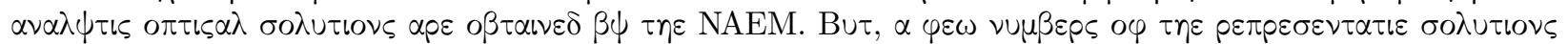

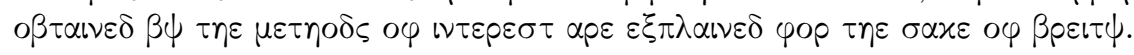

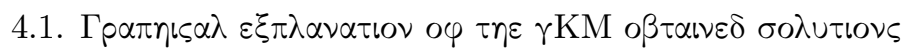

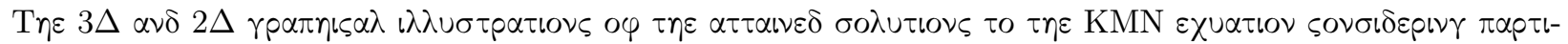

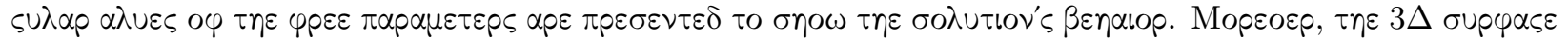




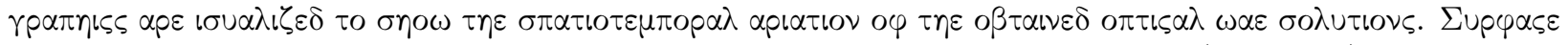

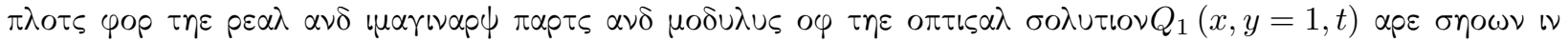

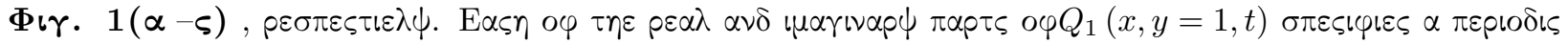

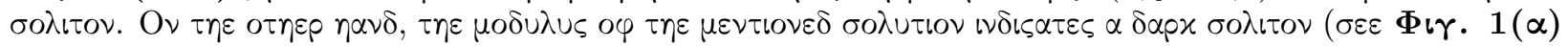

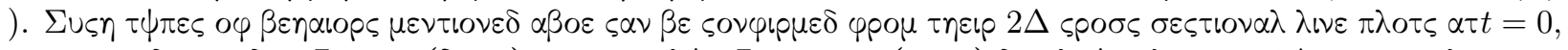

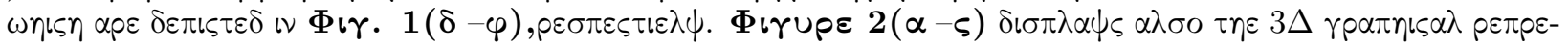

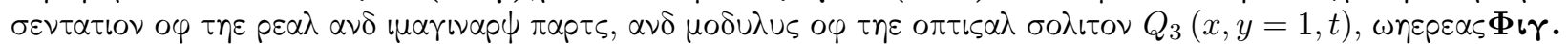

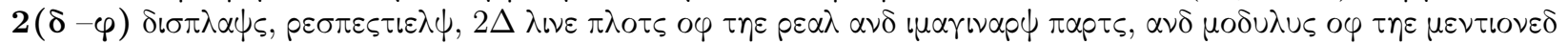

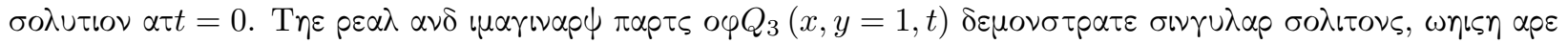

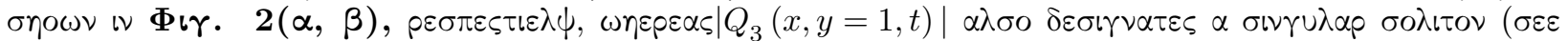

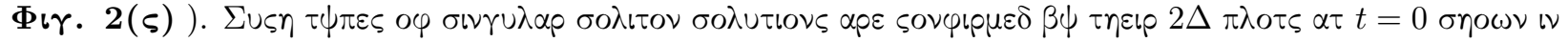

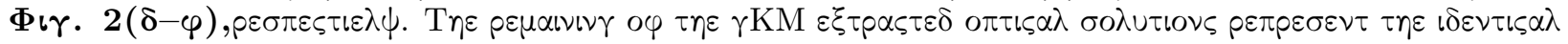

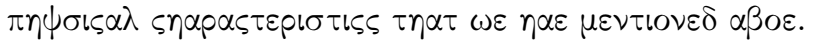

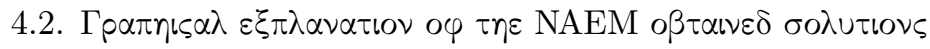

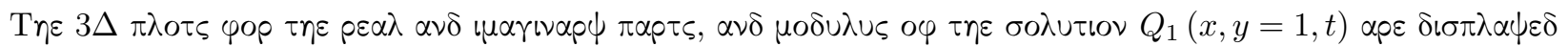

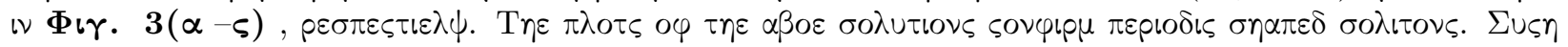

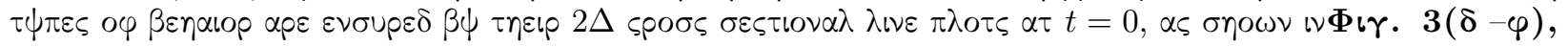

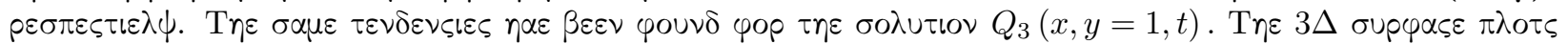

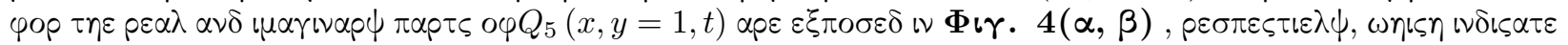

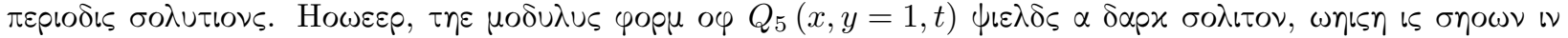

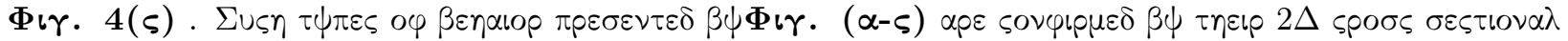

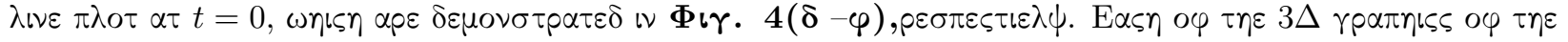

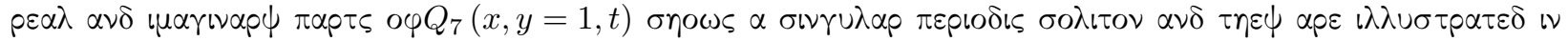

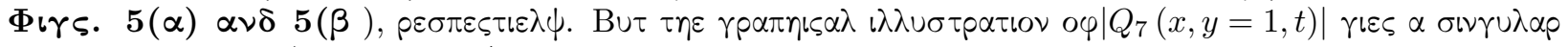

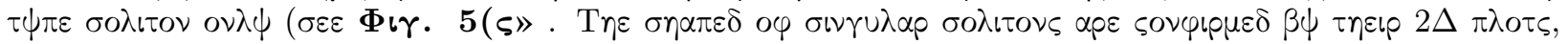

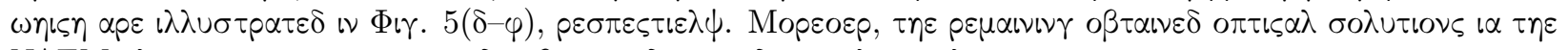

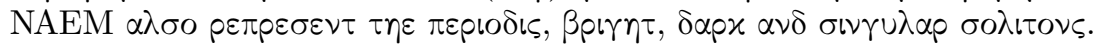

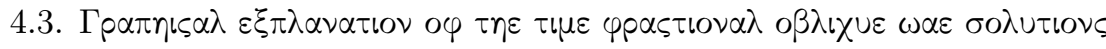

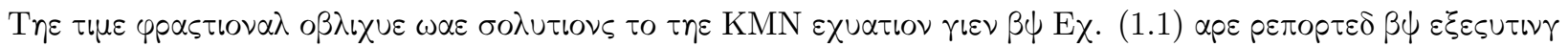

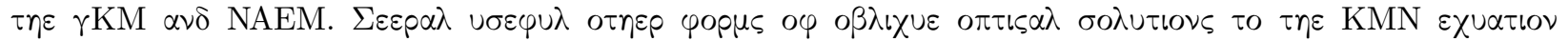

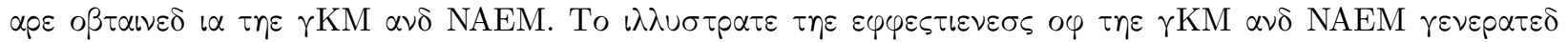

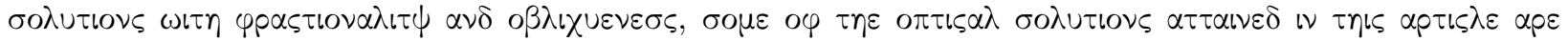

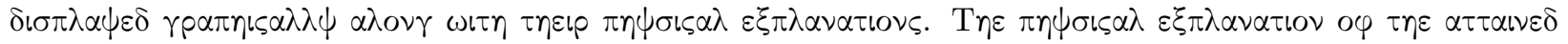

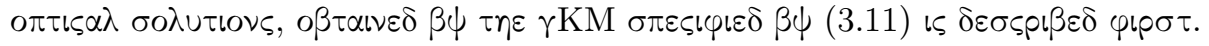

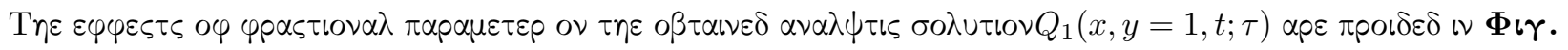

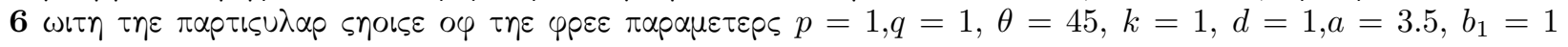

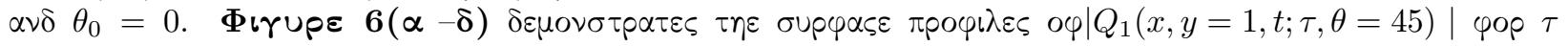

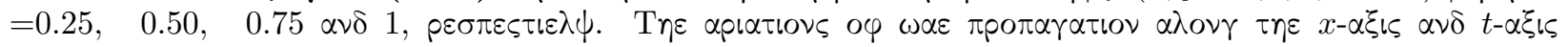

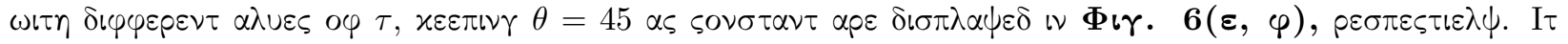

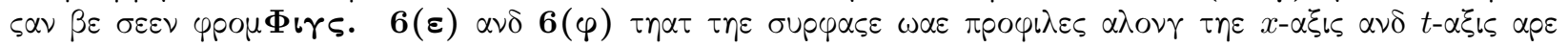

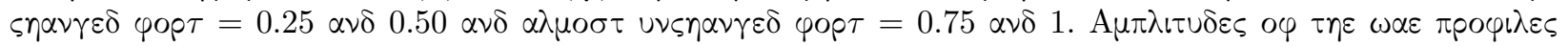

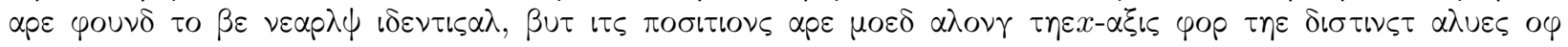
$\tau=0.25, \quad 0.50, \quad 0.75 \alpha \nu \delta 1$.

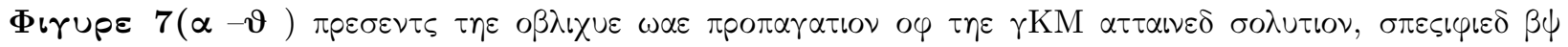

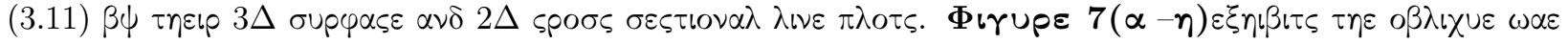

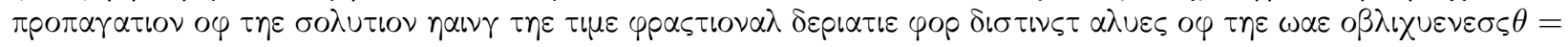

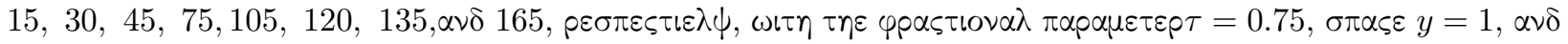

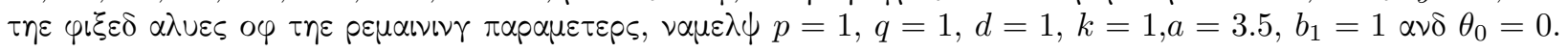




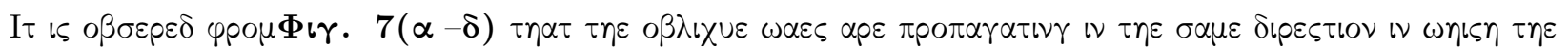

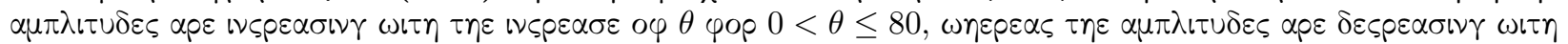

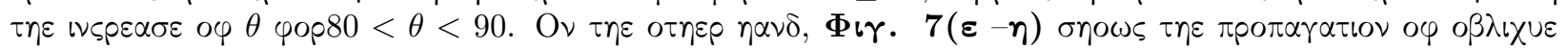

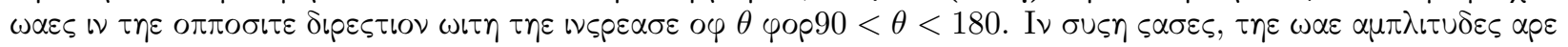

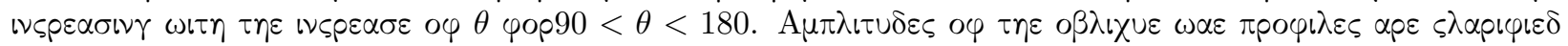

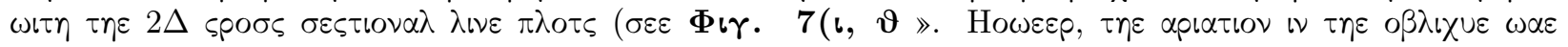

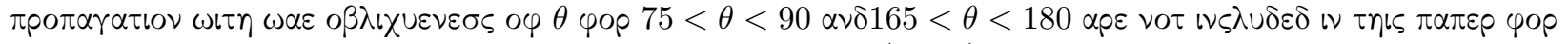

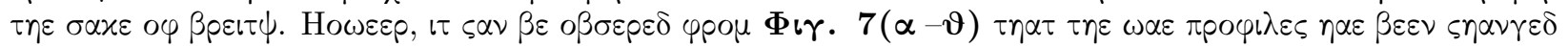

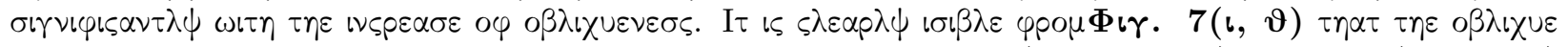

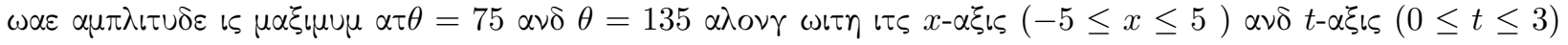

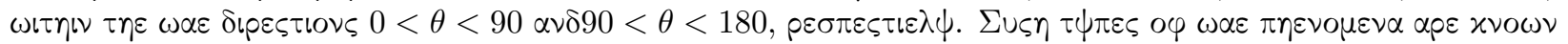

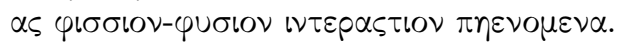

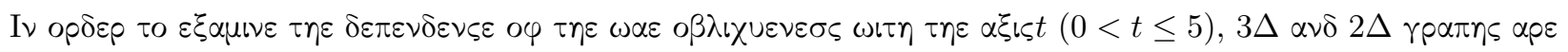

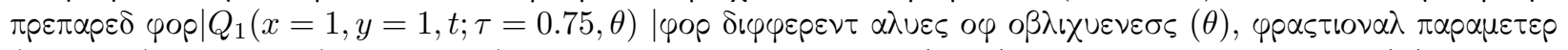

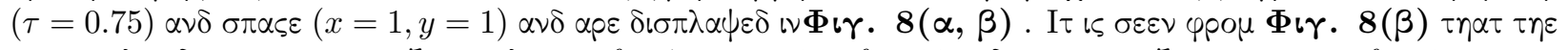

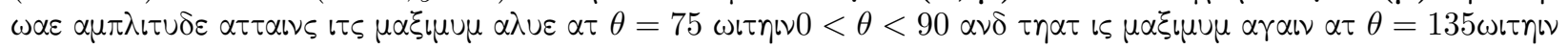

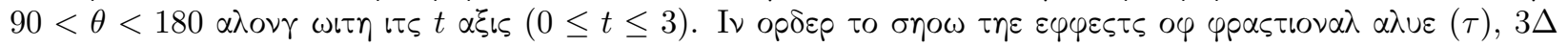

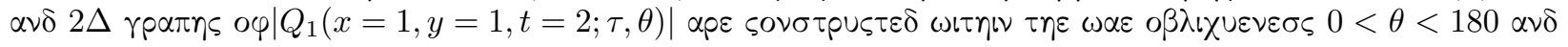

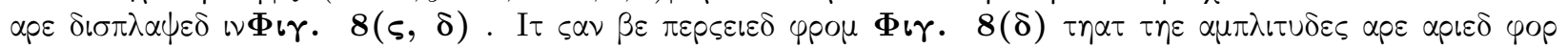

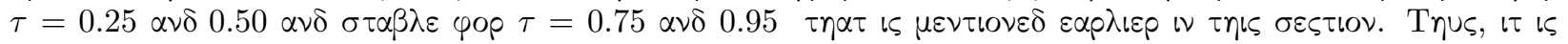

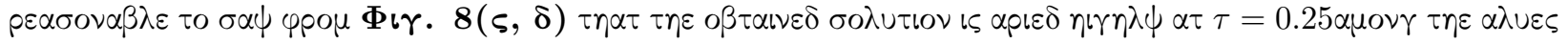

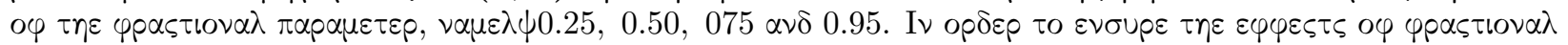

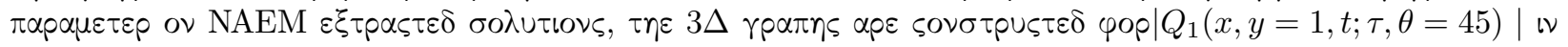

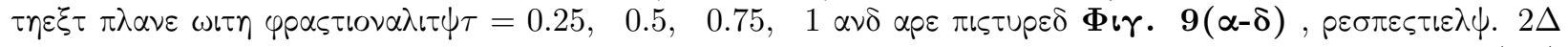

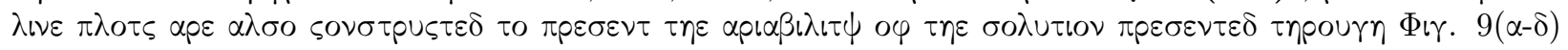

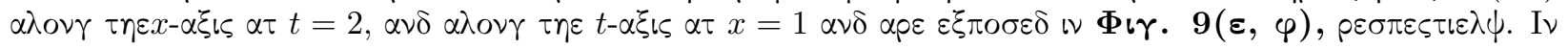

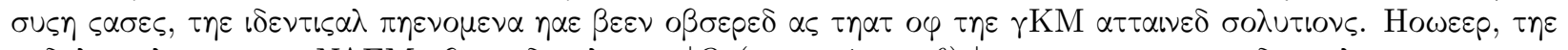

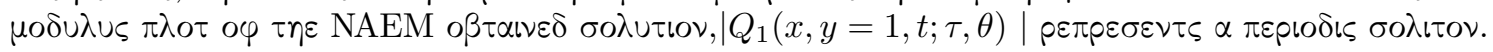

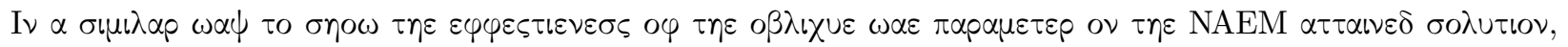

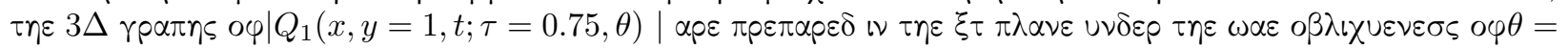

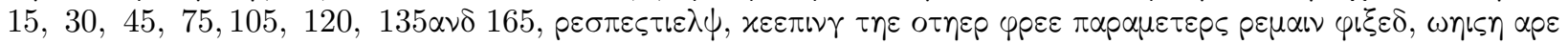

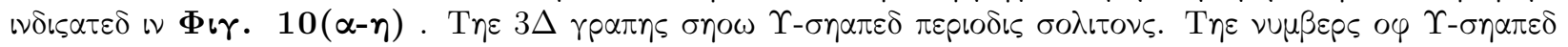

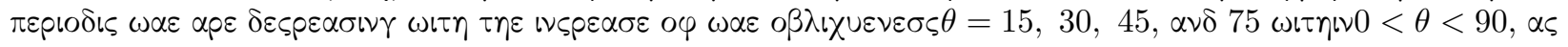

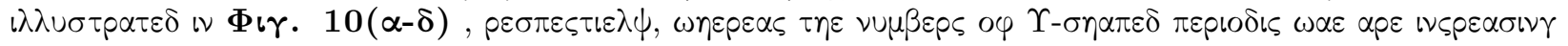

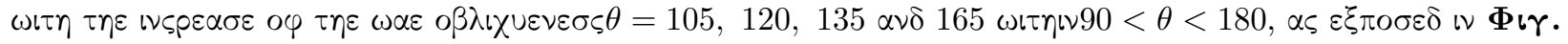

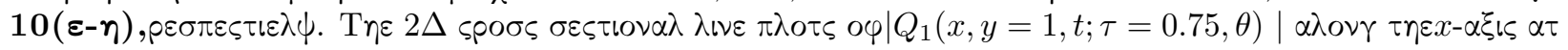

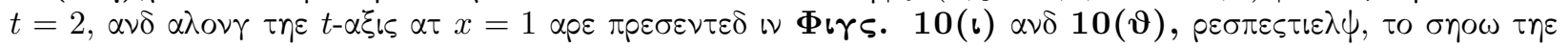

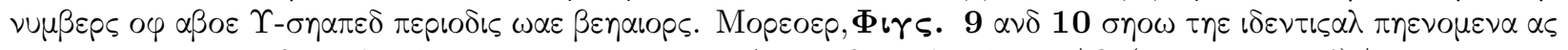

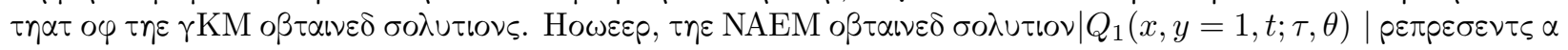

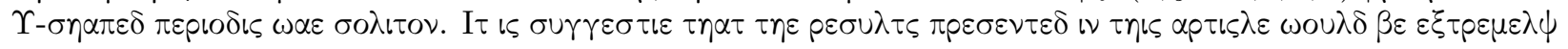

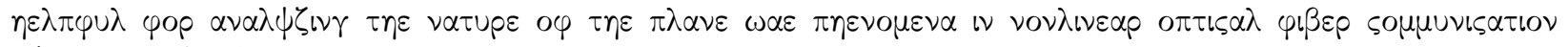

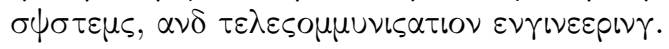

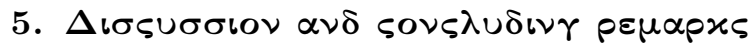

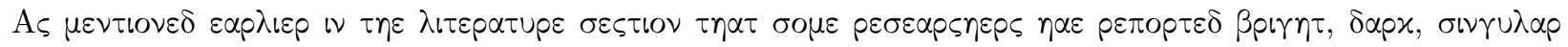

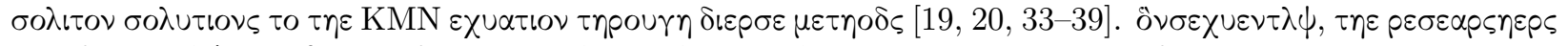

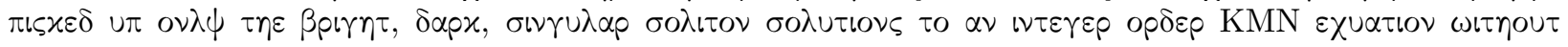

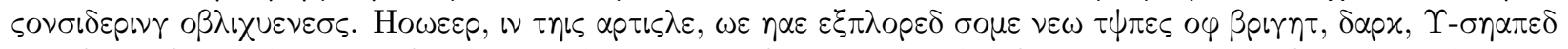

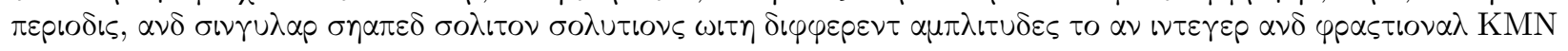




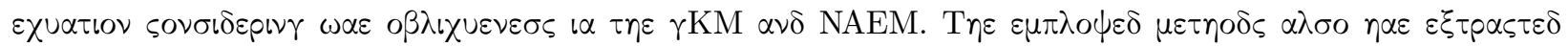

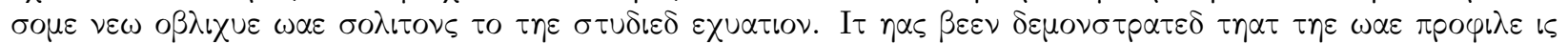

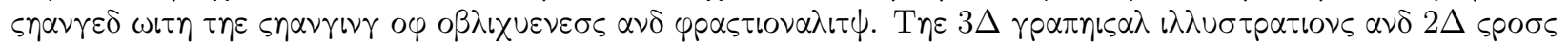

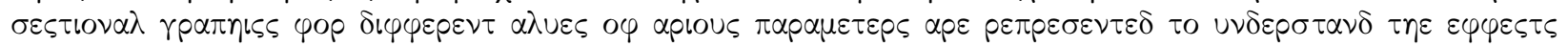

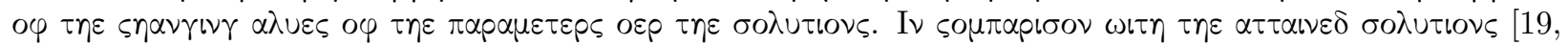

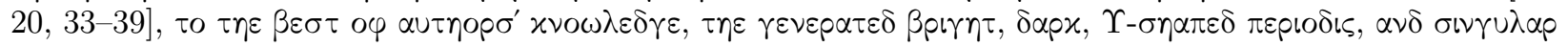

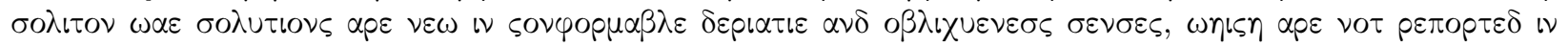

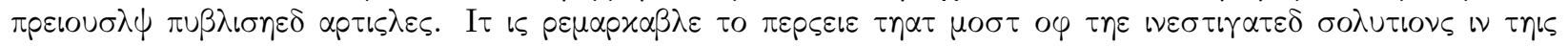

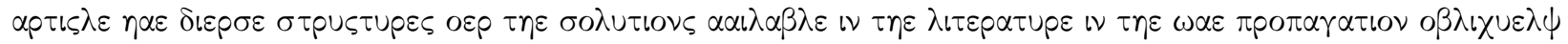

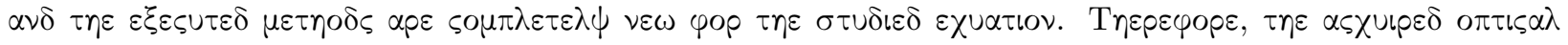

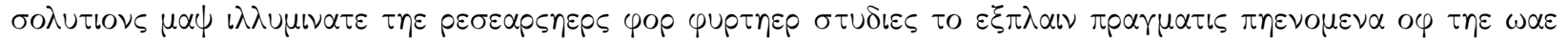

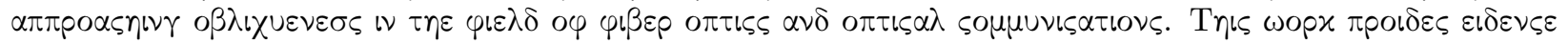

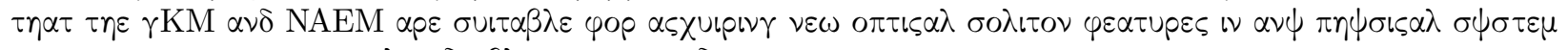

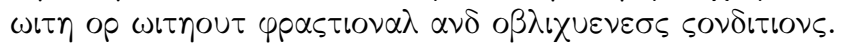

ö $\mu \pi \lambda \iota \alpha \nu \varsigma \varepsilon \omega \iota \tau \eta \varepsilon \tau \eta \iota \varsigma \alpha \lambda \sigma \tau \alpha \nu \delta \alpha \rho \delta \varsigma$

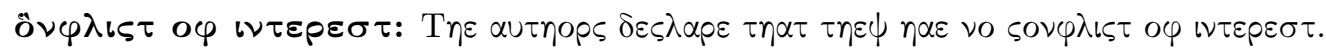

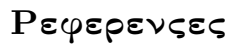

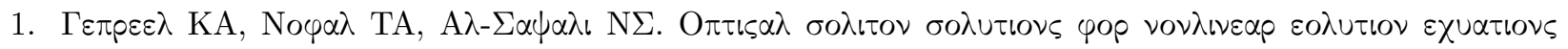

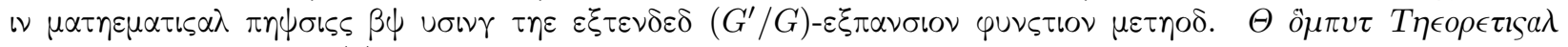

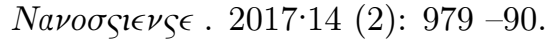

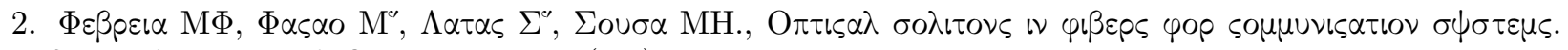

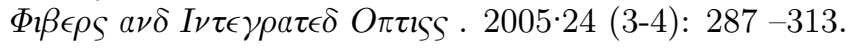

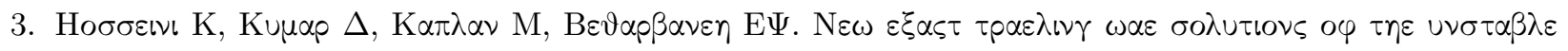

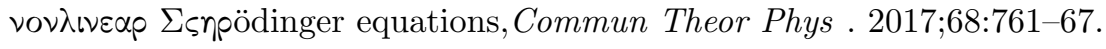

4. Xianguo X, Wu J. Riemann-Hilbert approach and N-soliton solutions for a generalized Sasa-Satsuma equation. Wave Motion . 2016;60: 62 -72.

5. Tasbozan O, Kurt A, Tozar A. New optical solutions of complex Ginzburg-Landau equation arising in semiconductor lasers. Applied Phys B . 2019;125(6): 104.

6. Biswas A, Alqahtani RT. Chirp-free bright optical solitons for perturbed Gerdjikov-Ivanov equation by semi-inverse variational principle, Optik 2017;147: $72-76$.

7. Bansal A, Biswas A, Triki H, Zhou Q, Moshokoa SP, Belic M. Optical solitons and group invariant solutions to Lakshmanan-Porsezian-Daniel model in optical fibers and PCF. Optik 2018;160: 86 -91.

8. Rezazadeh H, Kumar D, Neirameh A, Eslami M, Mirzazadeh M. Applications of three methods for obtaining optical soliton solutions for the Lakshmanan-Porsezian-Daniel model with Kerr law nonlinearity.Pramana 2019;94(1): 39.

9. Zhao Y, Fan E, N-soliton solution for a higher-order Chen-Lee-Liu equation with nonzero boundary conditions, Modern Physics Letters B . 2020;34(04): 2050054.

10. Triki H, Babatin MM, Biswas A. Chirped bright solitons for Chen-Lee-Liu equation in optical fibers and PCF. Optik2017;149: $300-303$.

11. Kudryashov NA. General solution of the traveling wave reduction for the perturbed Chen-Lee-Liu equation. Optik 2019;186: $339-49$.

12. Kumar D, Joardar AK, Hoque A, Paul GC. Investigation of dynamics of nematicons in liquid crystals by extended sinh-Gordon equation expansion method. Opt Quant Electron. 2019; 51(7): 212. 
13. Mirzazadeh M, Eslami M, Biswas A. Dispersive optical solitons by Kudryashov's method. Optik 2014;125(23): $6874-80$.

14. Zhou Q, Kumar D, Mirzazadeh M, Eslami M, Rezazadeh H. Optical soliton in nonlocal nonlinear medium with cubic-quintic nonlinearities and spatio-temporal dispersion. Acta Physica Polonica A . 2018;134(6): 1204 -10 .

15. Kumar D, Kaplan M. Application of the modified Kudryashov method to the generalized SchrödingerBoussinesq equations. Opt Quant Electron . 2018;50(9): 329.

16. Kaplan M, Hosseini K, Samadani F, Raza N. Optical soliton solutions of the cubic-quintic non-linear Schrödinger's equation including an anti-cubic term. J Modern Optics . 2018;65(12):1431-36.

17. Yasar E, Yıldırım Y, Adem AR. Perturbed optical solitons with spatio-temporal dispersion in (2+1)dimensions by extended Kudryashov method. Optik. 2018;158: 1-14.

18. Biswas A, Mirzazadeh M, Eslami M, Zhou Q, Bhrawy A, Belic M. Optical solitons in nanofibers with spatio-temporal dispersion by trial solution method. Optik . 2016;127(18): $7250-57$.

19. Ekici M, Sonmezoglu A, Biswas A, Belic MR. Optical solitons in (2+1)-dimensions with KunduMukherjee-Naskar equation by extended trial function scheme. Chinese J Phys . 2019;57: 72-77.

20. Yıldirım Y. Optical solitons to Kundu-Mukherjee-Naskar model with modified simple equation approach. Optik . 2019;184: 247-52.

21. Inc M, Aliyu AI, Yusuf A, Baleanu D. Optical solitons to the resonance nonlinear Schrödinger equation by sine-Gordon equation method.Superlattice Microst. 2018;113: 541-549.

22. Kumar D, Hosseini K, Samadani F. The sine-Gordon expansion method to look for the traveling wave solutions of the Tzitzéica type equations in nonlinear optics. Optik. 2017;149: 439-46.

23. Seadawy AR, Kumar D, Chakrabarty AK. Dispersive optical soliton solutions for the hyperbolic and cubic-quintic nonlinear Schrödinger equations via the extended sinh-Gordon equation expansion method.Eur Phys J Plus . 2018;133(5):182.

24. Bulut H, Sulaiman TA, Baskonus HM. Dark, bright and other soliton solutions to the Heisenberg ferromagnetic spin chain equation,Superlattice Microst. 2018;123: 12-19.

25. Kumar D, Manafian J, Hawlader F, Ranjbaran A. New closed form soliton and other solutions of the Kundu-Eckhaus equation via the extended sinh-Gordon equation expansion method. Optik. 2018;160: 159167.

26. Kudryashov NA. Simplest equation method to look for exact solutions of nonlinear differential equations. Chaos Solitons Fractal.2005;24(5): 1217-31.

27. Bilige S, Chaolu T, Wang X. Application of the extended simplest equation method to the coupled Schrödinger-Boussinesq equation. Appl Math Comput . 2013;224: 517-23.

28. Rezazadeh H, Mirhosseini-Alizamini SM, Eslami M, Rezazadeh M, Mirzazadeh M, Abbagari S. New optical solitons of nonlinear conformable fractional Schrödinger-Hirota equation. Optik. 2018;172: 545-53.

29. Khater MMA, Seadawy AR, Lu D. Dispersive optical soliton solutions for higher order nonlinear SasaSatsuma equation in mono mode fibers via new auxiliary equation method. Superlattice Microst. 2018;113: $346-58$.

30. Kundu A, Mukherjee A, Naskar T. Modelling rogue waves through exact dynamical lump soliton controlled by ocean currents. Proceedings of the Royal Society A. 2014;470: 20130576.

31. Qiu D, Zhang Y, He J. The rogue wave solutions of a new (2+1)-dimensional equation, Communs Nonlinear Sci Numel Simulation. 2016;30(1-3): 307-15. 
32. Kundu A, Mukherjee A. Novel integrable higher-dimensional nonlinear Schrodinger equation: properties, solutions, applications. arXiv . 2013; 1305.4023.

33. Yıldırım Y. Optical solitons to Kundu-Mukherjee-Naskar model with trial equation approach. Optik . 2019;183: 1061-65.

34. Aliyu AI, Li Y, Baleanu D. Single and combined optical solitons and conservation laws in $(2+1)-$ dimensions with Kundu-Mukherjee-Naskar equation. Chinese J Phys. 2020; 63: 410-18.

35. Yıldırım Y, Mirzazadeh M. Optical pulses with Kundu-Mukherjee-Naskar model in fiber communication systems. Chinese J Phys. 2020;64: 183-93.

36. Jhangeer A, Seadawy AR, Ali F, Ahmed A. New complex waves of perturbed Shrödinger equation with Kerr law nonlinearity and Kundu-Mukherjee-Naskar equation. Results Phys. 2020;16: 102816.

37. Biswas A, Guzman JV, Bansal A, Kara AH, Alzahrani AK, Zhou Q, Belic MR. Optical dromions, domain walls and conservation laws with $\mathrm{Kundu}-\mathrm{Mukherjee-Naskar}$ equation via traveling waves and Lie symmetry, Results Phys. 2020;16: 102850.

38. Yıldırım Y. Optical solitons to Kundu-Mukherjee-Naskar model in birefringent fibers with modified simple equation approach.Optik. 2019;184: 121-27.

39. Kudryashov NA. General solution of traveling wave reduction for the Kundu-Mukherjee-Naskar model. Optik . 2019;186: $22-27$.

40. Khalil R, Al Horani M, Yousef A, Sababheh M. A new definition of fractional derivative, J Comput Appl Math . 2014; 264: 65-70.

41. Abdeljawad T. On conformable fractional calculus. J Comput Appl Math . 2015;279, 57-66.

42. Kumar D, Seadawy AR, Joardar AK. Modified Kudryashov method via new exact solutions for some conformable fractional differential equations arising in mathematical biology. Chinese J Phys . 2018;56(1): $75-85$.

43. Kumar D, Darvishi MT, Joardar AK. Modified Kudryashov method and its application to the fractional version of the variety of Boussinesq-like equations in shallow water. Opt Quant Electron . 2018;50(3): 128.

44. Foroutan M, Kumar D, Manafian J, Hoque A. New explicit soliton and other solutions for the conformable fractional Biswas-Milovic equation with Kerr and parabolic nonlinearity through an integration scheme. Optik. 2018;170: 190 - 202 .

45. Ferdous F, Hafez MG, Biswas A, Ekici M, Zhou Q, Alfiras M, Moshokoa SP, Belic MR. Oblique resonant optical solitons with Kerr and parabolic law nonlinearities and fractional temporal evolution by generalizedexp (- $\Phi(\xi))$-expansion. Optik . 2019;178: 439-48.

46. Akther S, Hafez MG, Ferdous F. Oblique resonance wave phenomena for nonlinear coupled evolution equations with fractional temporal evolution, Eur Phys J Plus. 2019;134(9): 473.

47. Ferdous F, Hafez MG. Oblique closed form solutions of some important fractional evolution equations via the modified Kudryashov method arising in physical problems. J Ocean Eng Sci. 2018;3(3): 244-52.

\section{Figures caption}

Figure 1. 3D plots of the solution $Q_{1}(x, y=1, t)$, obtained by the gKM: (a) real part, (b) imaginary part, (c) modulus, and (d)-(f): the cross sectional 2D line plots of (a)-(c) at $t=0$, respectively, for the particular choice of the free parameters $p=1, q=1, h_{1}=2, h_{2}=1, l_{1}=1, l_{2}=2, d=1, a=3.5, b_{1}=1$ and $\theta_{0}=0$.

Figure 2. 3D plots of the solution $Q_{3}(x, y=1, t)$, obtained by the gKM: (a) real part, (b) imaginary part, (c) modulus, and (d)-(f): the cross sectional 2D line plots of (a)-(c) at $t=0$, respectively, with $p=1, q=1$, $h_{1}=2, h_{2}=1, l_{1}=1, l_{2}=2, d=1, a=3.5, b_{1}=1$ and $\theta_{0}=0$. 
Figure 3 . 3D plots of the solution $Q_{1}(x, y=1, t)$, obtained by the NAEM: (a) real part, (b) imaginary part, (c) modulus, and (d)-(f): the cross sectional 2D line plots of (a)-(c) at $t=0$, respectively, with $\alpha=1$, $\beta=1, \sigma=1, p=1, q=1, h_{1}=2, h_{2}=1, l_{1}=1, l_{2}=2$, and $\theta_{0}=0$.

Figure 4. 3D plots of the solution $Q_{5}(x, y=1, t)$, obtained by the NAEM: (a) real part, (b) imaginary part, (c) modulus, and (d)-(f): the cross sectional 2D line plots of (a)-(c) at $t=0$, respectively, with $\alpha=1$, $\beta=3, \sigma=1, p=1, q=1, h_{1}=2, h_{2}=1, l_{1}=1, l_{2}=2$, and $\theta_{0}=0$.

Figure 5. 3D plots of the solution $Q_{7}(x, y=1, t)$, obtained by the NAEM: (a) real part, (b) imaginary part, (c) modulus, and (d)-(f): the cross sectional 2D line plots of (a)-(c) at $t=0$, respectively, with $7 \alpha=1$, $\beta=3, \sigma=1, p=1, q=1, h_{1}=2, h_{2}=1, l_{1}=1, l_{2}=2$, and $\theta_{0}=0$.

Figure 6. Effects of the fractional parameter on $\left|Q_{1}(x, y=1, t ; \tau, \theta=45)\right|$, obtained by the gKM: (a)(d) with $\tau=0.25,0.50,0.75,1$, respectively, for the particular choice of the free parameters $p=1$, $q=1, \theta=45, d=1, k=1, a=3.5, b_{1}=1$ and $\theta_{0}=0$, and the cross sectional 2D line plots of (a)-(d): (e) variation of the surface profile along $x$-axis at $t=2$ and (f) variation of the surface profile along $t$-axis at $x=1$.

Figure 7. Effects of wave obliqueness on $\left|Q_{1}(x, y=1, t ; \tau=0.75, \theta)\right|$, obtained by the gKM: (a)-(h) of $\theta=$ $15,30,45,75,105,120,135,165$, respectively, with $p=1, q=1, \tau=0.75, d=1, k=1, a=3.5, b_{1}=1$, and $\theta_{0}=0$, and the cross sectional $2 \mathrm{D}$ line plots of (a)-(h): (i) variation of the surface profile along $x$-axis at $t=2$ and $(\mathrm{j})$ variation of the surface profile along $t$-axis at $x=1$.

Figure 8 . Effects of wave obliqueness on $\left|Q_{1}(x=1, y=1, t ; \tau=0.75, \theta)\right|$, obtained by the gKM: (a) 3D plot and (b) variation of the surface profile alongt-axis with respect to different oblique wave directions. Effects of the fractional parameter on the solution $\left|Q_{1}(x=1, y=1, t=2 ; \tau, \theta)\right|$, obtained by the gKM: (c) 3D plot and (d) variation of the surface profile along oblique wave direction with respect to different fractional values.

Figure 9 . Effects of the fractional parameter on $\left|Q_{1}(x, y=1, t ; \tau, \theta=45)\right|$, obtained by the NAEM: (a)-(d) of $\tau=0.25, \quad 0.5, \quad 0.75, \quad 1$, respectively, for the particular choice of the free parameters $\alpha=1, \beta=1, \sigma=1$, $p=1, q=1, k=1, \theta=45$ and $\theta_{0}=0$, and the cross sectional 2D line plots of (a)-(d): (e) variation of the surface profile along $x$-axis at $t=2$ and (f) variation of the surface along $t$-axis at $x=1$.

Figure 10 . Effects of wave obliqueness on $\left|Q_{1}(x, y=1, t ; \tau=0.75, \theta)\right|$, obtained by the NAEM: (a)-(h) of $\theta=15,30,45,75,105,120,135,165$, respectively, with $\alpha=1, \beta=1, \sigma=1, p=1, q=1, k=1, \tau=$ 0.75 and $\theta_{0}=0$, and the cross sectional 2D line plots of (a)-(h): (i) variation of the surface profile along $x$-axis at $t=2$ and $(\mathrm{j})$ variation of the surface profile along $t$-axis at $x=1$. 